\title{
R-parity violation and the cosmological gravitino problem
}

\author{
G. Moreau ${ }^{1}$ and M. Chemtob ${ }^{2}$ \\ 1: Centre de Physique Théorique, CNRS, Luminy, Case 907 \\ F-13288 Marseille Cedex 9, France \\ 2: Service de Physique Théorique, CE-Saclay \\ F-91191 Gif-sur-Yvette Cedex, France
}

October 31, 2018

\begin{abstract}
Based on the R-parity violation option of the minimal supersymmetric Standard Model, we examine the scenario where the massive gravitino, relic from the hot big-bang, is the lightest supersymmetric particle and can decay through one or several of the trilinear R-parity violating interactions. We calculate the rates of the gravitino decay via the various three-body decay channels with final states involving three quarks and/or leptons. By taking into account the present constraints on the trilinear R-parity violating coupling constants and assuming the gravitino and scalar superpartner masses do not exceed $\mathcal{O}(10 \mathrm{TeV})$, it turns out that the gravitinos could easily have decayed before the present epoch but not earlier than the big-bang nucleosynthesis one. Therefore, the considered scenario would upset the standard big-bang nucleosynthesis and we conclude that it does not seem to constitute a natural solution for the cosmological gravitino problem.
\end{abstract}

PACS numbers: 04.65+e, 95.30.Cq, 98.80.Ft, 12.60.Jv, 14.80.Ly

\section{Introduction}

In supergravity theories [1], the gravitino, namely the spin-3/2 supersymmetric partner of the graviton, weakly interacts with all the particle species (including itself) due to the small gravitational strength coupling $\sqrt{G_{N}}=1 / M_{P}, G_{N}$ being the gravitational constant and $M_{P}$ the Planck scale. Hence, the gravitino-gravitino annihilation rate is extremely small so that the gravitinos should decouple at an early epoch of the universe history, and moreover at an epoch characterized typically by a temperature $T$ higher than the gravitino mass: $k T>m_{3 / 2}$ ( $k$ being the Boltzmann constant) [2]. Therefore, the relic abundance of the gravitino should be large, which is often denoted in the literature as the cosmological "gravitino problem". One of the first solutions for the gravitino problem to be envisaged is to compensate the large gravitino relic abundance by a gravitino mass sufficiently small, namely $m_{3 / 2}<1 \mathrm{keV}$ [3], to respect the limit on the present universe energy density: $\Omega_{0} \lesssim 1$. For heavier gravitinos, a second type of available solution is by shortening their lifetime so that they do not survive out at the late epochs [2]. This can be realized in two characteristic options: either the gravitino is not the Lightest Supersymmetric Particle (LSP) and thus can decay into an odd number of superpartners through gravitational and gauge interactions (both of these ones couple an even number of superpartners), or 
it is the LSP. A gravitino LSP can be realized within various supersymmetric models, including the gauge mediated supersymmetry breaking models $[4,5]$, the models of low fundamental energy scale [6] and even the conventional gravity mediated supersymmetry breaking models (for some specific set of the supersymmetry breaking parameters). If the gravitino is the LSP, it can decay only into the ordinary particles of the Standard Model. Such a decay channel must involve [7] both gravitational and the so-called Rparity symmetry $[8,9]$ violating interactions (the latter ones couple an odd number of superpartners). The R-parity violating $\left(R_{p}\right)$ interactions are written in the following superpotential, in terms of the left-handed superfields for the leptons $(L)$, quarks $(Q)$ and Higgs of hypercharge $1 / 2(H)$ and the right-handed superfields for the charged leptons $\left(E^{c}\right)$, up and down type quarks $\left(U^{c}, D^{c}\right)$,

$$
W_{R_{p}}=\sum_{i, j, k}\left(\frac{1}{2} \lambda_{i j k} L_{i} L_{j} E_{k}^{c}+\lambda_{i j k}^{\prime} L_{i} Q_{j} D_{k}^{c}+\frac{1}{2} \lambda_{i j k}^{\prime \prime} U_{i}^{c} D_{j}^{c} D_{k}^{c}+\mu_{i} H L_{i}\right),
$$

$i, j, k$ being flavor indices, $\lambda_{i j k}, \lambda_{i j k}^{\prime}, \lambda_{i j k}^{\prime \prime}$ dimensionless coupling constants and $\mu_{i}$ dimension one parameters. Note that in the scenario in which the gravitino is not the LSP, the gravitino preferentially decays into an ordinary Standard Model particle and its superpartner through gravitational interactions, since the $R_{p}$ coupling constants are severely constrained by the low-energy experimental bounds obtained at colliders $[10,11]$.

In the scenario of an unstable gravitino heavier than the LSP, the gravitino decay can produce an unacceptable amount of LSP which conflicts with the observations of the present mass density of the universe [12]. The scenario containing an unstable LSP gravitino, having decay channels which involve $R_{p}$ coupling constants, is based on the violation of the R-parity symmetry. Now, neither the grand unified theories, the string theories nor the study of the discrete gauge symmetries give a strong theoretical argument in favor of the conservation of the R-parity symmetry in the supersymmetric extension of the Standard Model [10]. Hence, the scenario with an unstable LSP gravitino constitutes an attractive possibility which must be considered as an original potential solution with respect to the cosmological gravitino problem.

Our main purpose in the present work is to determine whether the scenario of an unstable LSP gravitino decaying via $R_{p}$ interactions constitutes effectively a natural solution to the cosmological gravitino problem. We will concentrate on the trilinear $\not R_{p}$ interactions, namely $\lambda_{i j k} L_{i} L_{j} E_{k}^{c}, \lambda_{i j k}^{\prime} L_{i} Q_{j} D_{k}^{c}$ and $\lambda_{i j k}^{\prime \prime} U_{i}^{c} D_{j}^{c} D_{k}^{c}$. With the goal of enhancing the gravitino instability, we will consider an optimistic type of scenario in which several trilinear $R_{p}$ coupling constants have simultaneously non-vanishing values. We will not consider the bilinear $R_{p}$ term $\mu_{i} H L_{i}$ of Eq.(1) which can always be rotated away by a suitable redefinition of superfields [13]. The bilinear $R_{p}$ interactions as well as the possible alternative of a spontaneous breaking of the R-parity symmetry have been considered, within the context of the cosmological gravitino problem, in a recent study [14] which examines the two-body gravitino decay mode into photon and neutrino (we will compare the results of [14] with ours). We also mention here the works [15] and [16] in which have been considered the $R_{p}$ decay reactions of single nucleon into a gravitino and a strange meson.

In Section 2, we determine the constraints arising from the cosmological gravitino problem in a scenario characterized by an unstable gravitino. In Section 3, we calculate the dominant gravitino decay rates involving the trilinear $R_{p}$ interactions. Finally, in Section 4 , we give a quantitative discussion aimed at determining whether the scenario of an unstable LSP gravitino decaying through $R_{p}$ interactions can effectively constitute 
a natural solution with regard to the cosmological gravitino problem. This quantitative discussion is based on the results of Sections 2 and 3 as well as the present low-energy constraints on the $R_{p}$ coupling constants [11].

\section{Constraints on the gravitino decay temperature}

In this section, we derive the constraints [2] characteristic of a scenario containing an unstable gravitino supposed to solve the cosmological gravitino problem.

At the temperature $k T \approx m_{3 / 2}$, the expansion rate of the universe is $H\left(k T \approx m_{3 / 2}\right) \approx$ $\sqrt{m_{3 / 2}}(k T)^{3 / 2} / M_{P}=m_{3 / 2}^{2} / M_{P}$. Besides, the gravitino decay having the larger rate is the two-body decay into a particle of the Standard Model and its supersymmetric partner. The largest gravitino decay rate is thus of order,

$$
\Gamma_{\max } \sim m_{3 / 2}^{3} / M_{P}^{2}
$$

which is smaller than $H\left(k T \approx m_{3 / 2}\right)$ by a factor $M_{P} / m_{3 / 2}$. Therefore, the equilibrium condition,

$$
\Gamma \geq H(T)
$$

$\Gamma$ being the gravitino decay rate, can only be reached for temperatures much smaller than $k T \approx m_{3 / 2}$. In other words, the gravitinos cannot decay as long as the temperature has not dropped far below the gravitino mass. This conclusion has two important consequences.

The first consequence is that the gravitino decays occur at temperatures at which the gravitinos cannot be created in collisions, so that the gravitino population can effectively be reduced by the gravitino decays.

The other consequence is that the equilibrium condition (Eq.(3)) is reached at an epoch at which the cosmic energy density is dominated by the gravitino energy density $\rho_{3 / 2}$. Therefore, in determining the temperature $T_{3 / 2}$ at which the equilibrium condition of Eq.(3) is reached (the gravitino decay temperature) by solving the equation $\Gamma=H\left(T_{3 / 2}\right)$, one must take the following expression for the expansion rate,

$$
H(T) \approx \sqrt{\frac{8 \pi G_{N}}{3} \rho_{3 / 2}} .
$$

Since the gravitino mass density reads as,

$$
\rho_{3 / 2}=\frac{3 \zeta(3)}{\pi^{2}} \frac{g(T)}{g\left(T_{d}\right)} m_{3 / 2}(k T)^{3},
$$

where $\zeta(x)$ is the Riemann zeta function and $\zeta(3)=1.20206 \ldots$, the equation Eq.(4) can be rewritten as,

$$
H(T) \approx \sqrt{\frac{8 \zeta(3)}{\pi} \frac{g(T)}{g\left(T_{d}\right)}} \frac{\sqrt{m_{3 / 2}}}{M_{P}}(k T)^{3 / 2} .
$$

In Eq.(5) and Eq.(6), g(T) counts the total number of effectively massless degrees of freedom (those of species with mass $m \ll k T$ ), and $T_{d}$ is the gravitino decoupling temperature, namely the temperature at which is reached the out of equilibrium condition $\Gamma_{a n}<H(T), \Gamma_{a n}$ being the gravitino-gravitino annihilation rate. The gravitino decoupling temperature is typically larger than the gravitino mass, as we have mentioned in 
the introduction. The presence of the factor $g(T) / g\left(T_{d}\right)$ in Eq.(5) and Eq.(6) allows to take into account the increase of the temperature due to the conservation of the entropy per comoving volume $S=\left(2 \pi^{2} / 45\right) g(T)(k T)^{3} R^{3}$, where $R=R(t)$ is the time dependent scale factor. From Eq.(6) it follows that the gravitino decay temperature is given by,

$$
k T_{3 / 2} \approx\left(\frac{\pi}{8 \zeta(3)} \frac{g\left(T_{d}\right)}{g\left(T_{3 / 2}\right)}\right)^{1 / 3}\left(\frac{\Gamma^{2} M_{P}^{2}}{m_{3 / 2}}\right)^{1 / 3} .
$$

At the temperature $T_{3 / 2}$, most of the gravitinos decay. After the gravitinos have decayed and their decay energy has been thermalized, the temperature rises to the value $k T_{3 / 2}^{\prime}$ given by,

$$
k T_{3 / 2}^{\prime} \approx \frac{(90 \zeta(3))^{1 / 4}}{\pi}\left(\frac{m_{3 / 2}\left(k T_{3 / 2}\right)^{3}}{g\left(T_{3 / 2}\right)}\right)^{1 / 4} .
$$

The equation Eq.(8) is nothing else but the expression of the energy density conservation. From Eq.(7) and Eq.(8) it follows that $k T_{3 / 2}^{\prime}$ reads as,

$$
k T_{3 / 2}^{\prime} \approx\left(\frac{45}{4 \pi^{3}}\right)^{1 / 4} \frac{g\left(T_{d}\right)^{1 / 4}}{g\left(T_{3 / 2}\right)^{1 / 2}} \sqrt{\Gamma M_{P}} .
$$

In order to avoid large relic abundance of an unstable gravitino, this one must decay before the present epoch. This requirement imposes the following bound on the increased gravitino decay temperature $T_{3 / 2}^{\prime}$,

$$
T_{3 / 2}^{\prime}>2.75 \mathrm{~K} \quad\left(k T_{3 / 2}^{\prime}>2.3610^{-10} \mathrm{MeV}\right) .
$$

Furthermore, the decay of the gravitinos leads to an increase of the temperature by a factor which can be deduced from Eq.(7) and Eq.(9):

$$
\begin{aligned}
\frac{T_{3 / 2}^{\prime}}{T_{3 / 2}} & \approx \frac{45^{1 / 4} \sqrt{2} \zeta(3)^{1 / 3}}{\pi^{13 / 12}}\left(g\left(T_{3 / 2}\right) \sqrt{g\left(T_{d}\right)}\right)^{-1 / 6}\left(\frac{m_{3 / 2}}{\sqrt{\Gamma M_{P}}}\right)^{1 / 3} \\
& >\frac{45^{1 / 4} \sqrt{2} \zeta(3)^{1 / 3}}{\pi^{13 / 12}}\left(g\left(T_{3 / 2}\right) \sqrt{g\left(T_{d}\right)}\right)^{-1 / 6}\left(\frac{M_{P}}{m_{3 / 2}}\right)^{1 / 6} .
\end{aligned}
$$

In order to derive the bound of Eq.(11), we have used the fact that $\Gamma<\Gamma_{\max }$ (see Eq.(2)). This increase of the temperature leads to an increase of the entropy density $s=\left(2 \pi^{2} / 45\right) g(T)(k T)^{3}$ and hence to a decrease of the baryon-to-entropy ratio (or baryon number in a comoving volume) $B=n_{B} / s, n_{B}$ being the baryon number density. Therefore, if the gravitinos decay after the nucleosynthesis epoch, the baryon-to-entropy ratio during the nucleosynthesis epoch must be much greater than the present one, leading to the production through nucleosynthesis of too much helium and too little deuterium (compared with the constraints on the primordial abundances derived from observational data) [2]. In conclusion, if the gravitinos decay before the present epoch, those decays have to occur before the nucleosynthesis epoch, or in other words, the increased gravitino decay temperature $T_{3 / 2}^{\prime}$ has to be higher than the nucleosynthesis temperature, namely,

$$
k T_{3 / 2}^{\prime}>0.4 M e V
$$

In summary, in a scenario of unstable gravitino protected against large gravitino relic abundance, the gravitino must decay before the present epoch, namely $k T_{3 / 2}^{\prime}>$ 
$2.3610^{-10} \mathrm{MeV}$ (see Eq.(10)), or even at an earlier epoch than the nucleosynthesis one, namely $k T_{3 / 2}^{\prime}>0.4 \mathrm{MeV}$ (see Eq.(12)), if one requires that this scenario does not conflict with the current cosmological ideas on nucleosynthesis. Hence, in order to determine whether a given scenario of unstable gravitino can effectively constitute a solution to the gravitino problem, one has to calculate, within this scenario, the gravitino decay rate. Indeed, the value of the gravitino decay rate gives, through Eq.(9), the gravitino decay temperature $T_{3 / 2}^{\prime}$, and thus allows to verify whether the constraints of Eq.(10) and Eq.(12) are respected or not. The calculation of the gravitino decay rate will be done in the next section, within the scenario we consider.

\section{Rates of the gravitino decay via trilinear $R$-parity violating interactions}

As we have discussed in the introduction, in the scenario we study, the gravitino is the LSP, so that it can decay only via $R_{p}$ interactions, and the R-parity symmetry is violated only by the trilinear terms of the superpotential 1 . Hence, in our scenario, the gravitino can only decay through the trilinear $\not k_{p}$ couplings of Eq.(1). The gravitino decay processes which involve the trilinear $R_{p}$ interactions of Eq.(1) and have the dominant rates are obviously the decays through a virtual scalar superpartner into three ordinary fermions involving one gravitational and one $R_{p}$ coupling. Those processes are represented in Fig.1, Fig.2 and Fig.3 for the $R_{p}$ interactions of type $\lambda_{i j k} L_{i} L_{j} E_{k}^{c}, \lambda_{i j k}^{\prime} L_{i} Q_{j} D_{k}^{c}$ and $\lambda_{i j k}^{\prime \prime} U_{i}^{c} D_{j}^{c} D_{k}^{c}$ (see Eq.(1)), respectively. The Feynman diagrams of Fig.1, Fig.2 and Fig.3 have been derived from the structures of the relevant interaction lagrangians. Let us collect these relevant interaction lagrangians. First, the locally supersymmetric lagrangian term which describes the coupling between the fields of the gravitino $\left(\Psi_{\mu}\right)$, an ordinary fermion $(\psi)$ and its scalar superpartner $(\phi)$ reads as,

$$
\mathcal{L}=-\frac{1}{\sqrt{2} M_{\star}} \bar{\psi}_{L} \gamma^{\mu} \gamma^{\nu} \partial_{\nu} \phi \Psi_{\mu R}+\text { h.c. },
$$

the $L / R$ indices standing for Left/Right chirality, $\gamma^{\mu}$ being the Dirac matrices and $M_{\star}=$ $\left(8 \pi G_{N}\right)^{-1 / 2}=2.410^{18} \mathrm{GeV}$ the reduced Planck mass. With our conventions, the reduced Planck mass $M_{\star}$ and the gravitino mass $m_{3 / 2}$ are related through the formula,

$$
m_{3 / 2}=\frac{F}{\sqrt{3} M_{\star}},
$$

where $F$ denotes the scale of spontaneous supersymmetry breaking. Secondly, the relevant lagrangian of the $\mathbb{R}_{p}$ interactions of type $\lambda, \lambda^{\prime}$ and $\lambda^{\prime \prime}$, originating from the superpotential 1 , is the following,

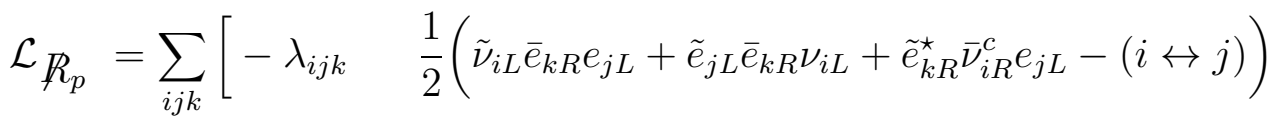

$$
\begin{aligned}
& -\lambda_{i j k}^{\prime} \quad\left(\tilde{\nu}_{i L} \bar{d}_{k R} d_{j L}+\tilde{d}_{j L} \bar{d}_{k R} \nu_{i L}+\tilde{d}_{k R}^{\star} \bar{\nu}_{i R}^{c} d_{j L}\right. \\
& \left.-\tilde{e}_{i L} \bar{d}_{k R} u_{j L}-\tilde{u}_{j L} \bar{d}_{k R} e_{i L}-\tilde{d}_{k R}^{\star} \bar{e}_{i R}^{c} u_{j L}\right) \\
& \left.-\lambda_{i j k}^{\prime \prime} \quad \frac{1}{2}\left(\tilde{u}_{i R}^{\star} \bar{d}_{j R} d_{k L}^{c}+2 \tilde{d}_{j R}^{\star} \bar{u}_{i R} d_{k L}^{c}\right)\right]+ \text { h.c., }
\end{aligned}
$$




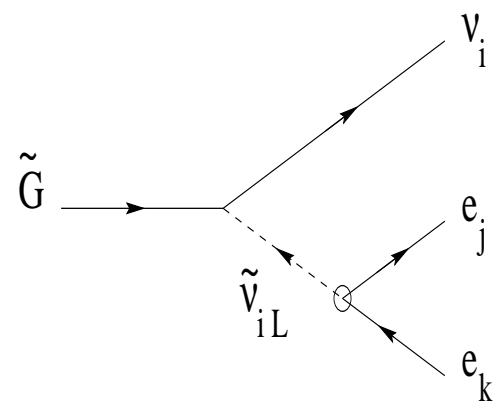

(a)

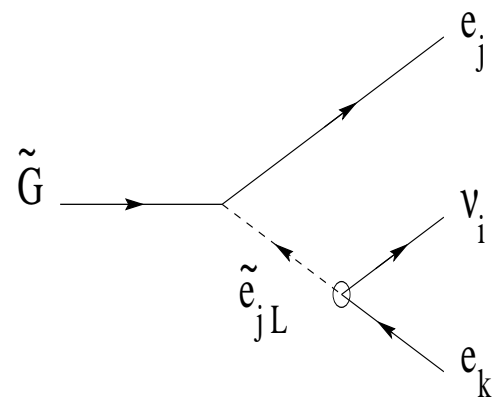

(b)

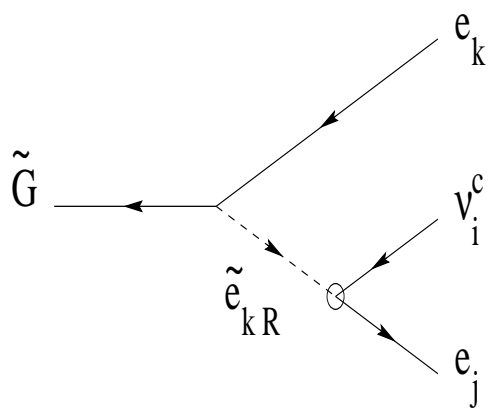

(c)

Figure 1: Feynman diagrams of the gravitino three-body decay processes involving the $\lambda_{i j k} L_{i} L_{j} E_{k}^{c}$ interactions. These interactions are represented by the circled vertex. The other vertex correspond to gravitational couplings. The plain (dashed) lines symbolize the propagation of fermionic (scalar) particles. The arrows denote the flow of momentum of the associated particles. Finally, $e, \tilde{e}, \nu, \tilde{\nu}$ and $\tilde{G}$ represent respectively the charged leptons, the charged sleptons, the neutrinos, the sneutrinos and the gravitino, $i, j, k$ are generation indices, the $L, R$ indices stand for Left, Right (chirality) and the $c$ exponent indicates a charge conjugated particle. We have not drawn the charge conjugated reactions which can be trivially deduced from these ones.

where $e, \nu, d$ and $u$ represent respectively the fields of the charged leptons, the neutrinos, the down and the up quarks, a indicates the scalar superpartner and our notation is such that one has, for instance, $\bar{\nu}_{i R}^{c}=\overline{\left(\nu_{i}^{c}\right)_{R}}$.

As shown by Fig.1, when the gravitino decays via a $\lambda_{i j k}$ coupling, it decays into two charged leptons and a neutrino as $\tilde{G} \stackrel{\lambda_{i j k}}{\rightarrow} \nu_{i} e_{j} \bar{e}_{k}, \bar{\nu}_{i} \bar{e}_{j} e_{k}$. Based on the lagrangians of Eq.(13) and Eq.(15), we have calculated the rate of this gravitino decay reaction, which receives three contributions as can be observed in Fig.1. The full analytical result for the spin summed amplitude of this gravitino decay reaction is given in Appendix B. We give here the analytical result for the integrated rate of the gravitino decay $\tilde{G} \stackrel{\lambda_{i j k}}{\rightarrow} \nu_{i} e_{j} \bar{e}_{k}$, under the assumption that the masses of the final state particles are negligible with respect to the gravitino and exchanged scalar superpartner masses (we will comment on this hypothesis below),

$$
\begin{aligned}
\Gamma\left(\tilde{G}^{\lambda_{i j k}} \nu_{i} e_{j} \bar{e}_{k}\right)= & \frac{1}{96(2 \pi)^{3}} \frac{\lambda_{i j k}^{2}}{m_{3 / 2}^{3} M_{\star}^{2}}\left[\Omega\left(m_{\tilde{\nu}_{i L}}\right)+\Omega\left(m_{\tilde{e}_{j L}}\right)+\Omega\left(m_{\tilde{e}_{k R}}\right)+\Xi\left(m_{\tilde{\nu}_{i L}}, m_{\tilde{e}_{k R}}\right)\right. \\
& +\Xi\left(m_{\tilde{e}_{j L}}, m_{\tilde{e}_{k R}}\right)+\Xi\left(m_{\tilde{\nu}_{i L}}, m_{\tilde{e}_{j L}}\right)+\Sigma\left(m_{\tilde{\nu}_{i L}}, m_{\tilde{e}_{k R}}\right)+\Sigma\left(m_{\tilde{e}_{j L}}, m_{\tilde{e}_{k R}}\right) \\
& +\Sigma\left(m_{\tilde{\nu}_{i L}}, m_{\tilde{e}_{j L}}\right)+\Sigma\left(m_{\tilde{e}_{j L}}, m_{\tilde{\nu}_{i L}}\right)+\Delta\left(m_{\tilde{\nu}_{i L}}, m_{\tilde{e}_{k R}}\right) \\
& {\left[S p\left(\frac{m_{\tilde{\nu}_{i L}}^{2}}{m_{\tilde{\nu}_{i L}}^{2}+m_{\tilde{e}_{k R}}^{2}-m_{3 / 2}^{2}}\right)-S p\left(\frac{m_{\tilde{\nu}_{i L}}^{2}-m_{3 / 2}^{2}}{m_{\tilde{\nu}_{i L}}^{2}+m_{\tilde{e}_{k R}}^{2}-m_{3 / 2}^{2}}\right)\right]+\Delta\left(m_{\tilde{e}_{j L}}, m_{\tilde{e}_{k R}}\right) } \\
& {\left[S p\left(\frac{m_{\tilde{e}_{j L}}^{2}}{m_{\tilde{e}_{j L}}^{2}+m_{\tilde{e}_{k R}}^{2}-m_{3 / 2}^{2}}\right)-S p\left(\frac{m_{\tilde{e}_{j L}}^{2}-m_{3 / 2}^{2}}{m_{\tilde{e}_{j L}}^{2}+m_{\tilde{e}_{k R}}^{2}-m_{3 / 2}^{2}}\right)\right]+\Delta\left(m_{\tilde{\nu}_{i L}}^{2}, m_{\tilde{e}_{j L}}^{2}\right) } \\
& {\left.\left[-S p\left(-\frac{m_{\tilde{e}_{j L}}^{2}}{m_{\tilde{\nu}_{i L}}^{2}}\right)-S p\left(-\frac{m_{\tilde{\nu}_{i L}}^{2}}{m_{\tilde{e}_{j L}}^{2}}\right)+S p\left(\frac{m_{3 / 2}^{2}-m_{\tilde{e}_{j L}}^{2}}{m_{\tilde{\nu}_{i L}}^{2}}\right)+S p\left(\frac{m_{3 / 2}^{2}-m_{\tilde{\nu}_{i L}}^{2}}{m_{\tilde{e}_{j L}}^{2}}\right)\right]\right], }
\end{aligned}
$$

where the mass functions $\Omega(m), \Xi\left(m_{1}, m_{2}\right), \Delta\left(m_{1}, m_{2}\right)$ and $\Sigma\left(m_{1}, m_{2}\right)$ are defined in Appendix A, $S p(x)=\operatorname{Polylog}(2, x)=L i_{2}(x)$ is the dilogarithm or Spence function and $m_{\tilde{\nu}_{i L}}, m_{\tilde{e}_{j L}}$ and $m_{\tilde{e}_{k R}}$ are respectively the masses of the superpartners $\tilde{\nu}_{i L}, \tilde{e}_{j L}$ and $\tilde{e}_{k R}$. 


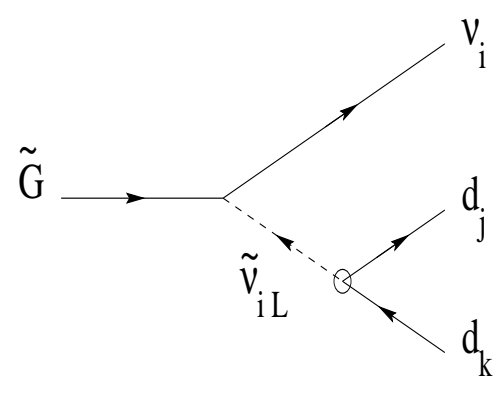

(a)

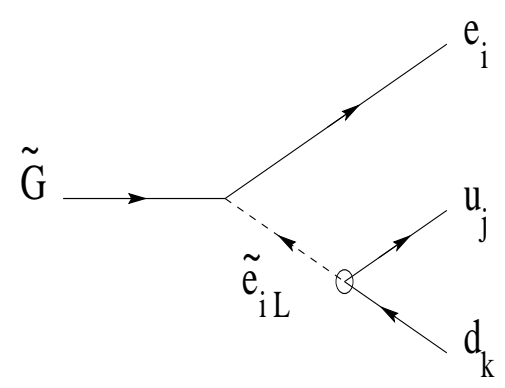

(d)

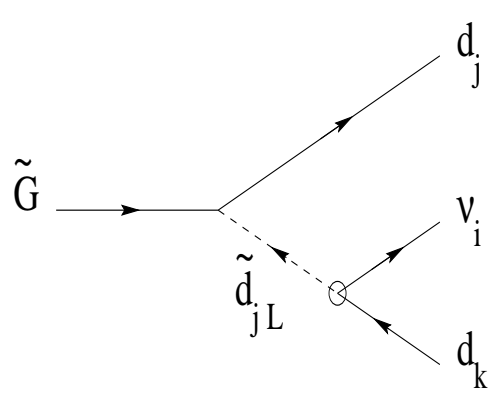

(b)

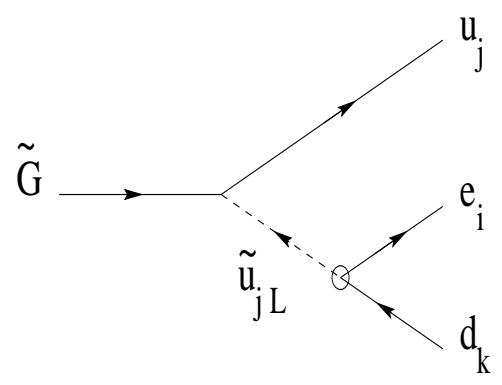

(e)

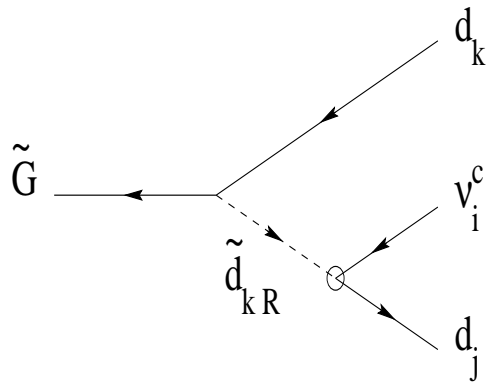

(c)

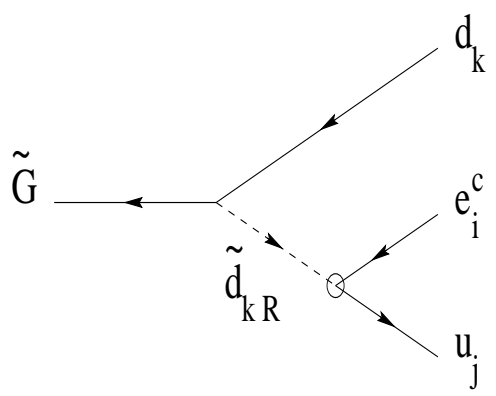

(f)

Figure 2: Feynman diagrams of the gravitino three-body decay processes involving the $\lambda_{i j k}^{\prime} L_{i} Q_{j} D_{k}^{c}$ interactions. These interactions are represented by the circled vertex. The other vertex correspond to gravitational couplings. The plain (dashed) lines symbolize the propagation of fermionic (scalar) particles. The arrows denote the flow of momentum of the associated particles. Finally, $e, \tilde{e}, \nu, \tilde{\nu}, d, \tilde{d}, u, \tilde{u}$ and $\tilde{G}$ represent respectively the charged leptons, the charged sleptons, the neutrinos, the sneutrinos, the down quarks, the down squarks, the up quarks, the up squarks and the gravitino, $i, j, k$ are generation indices, the $L, R$ indices stand for Left, Right (chirality) and the $c$ exponent indicates a charge conjugated particle. We have not drawn the charge conjugated reactions which can be trivially deduced from these ones.

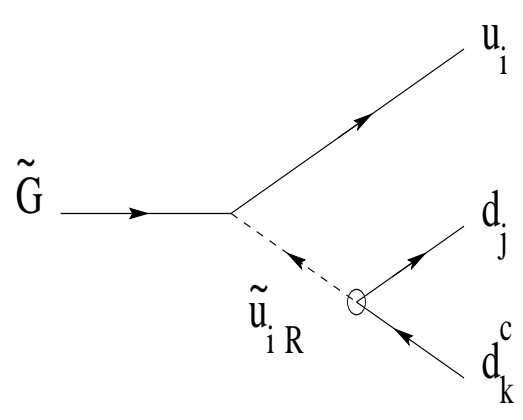

(a)

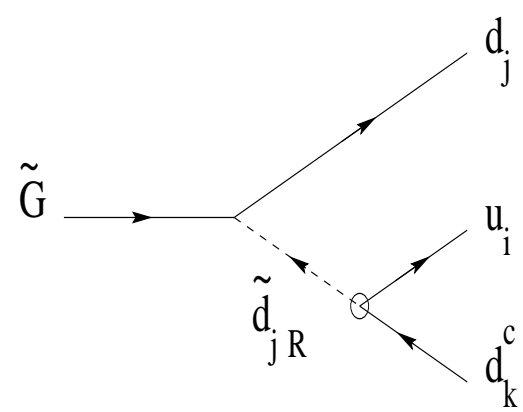

(b)

Figure 3: Feynman diagrams of the gravitino three-body decay processes involving the $\lambda_{i j k}^{\prime \prime} U_{i}^{c} D_{j}^{c} D_{k}^{c}$ interactions. These interactions are represented by the circled vertex. The other vertex correspond to gravitational couplings. The plain (dashed) lines symbolize the propagation of fermionic (scalar) particles. The arrows denote the flow of momentum of the associated particles. Finally, $d, \tilde{d}, u, \tilde{u}$ and $\tilde{G}$ represent respectively the down quarks, the down squarks, the up quarks, the up squarks and the gravitino, $i, j, k$ are generation indices, the $L, R$ indices stand for Left, Right (chirality) and the $c$ exponent indicates a charge conjugated particle. We have not drawn the charge conjugated reactions which can be trivially deduced from these ones. 
The hypothesis that the masses of the final state particles, in the reaction $\tilde{G} \stackrel{\lambda_{i j k}}{\rightarrow} \nu_{i} e_{j} \bar{e}_{k}$, are negligible with respect to the gravitino and exchanged scalar superpartner masses has been made in order to obtain a more simple form for the analytical result of the integrated gravitino decay rate (see Eq.(16)). Nevertheless, in Section 4, the numerical results will take into account the full mass effect of the final state particles. Moreover, this assumption that the masses of the final state particles are negligible with respect to the gravitino and exchanged scalar superpartner masses represents a good approximation justified by the two following points. First, the present experimental limits on the scalar superpartner masses (noted generically by $\tilde{m}$ ), obtained at colliders within an $R_{p}$ model containing a non-vanishing Yukawa coupling of type $\lambda, \lambda^{\prime}$ or $\lambda^{\prime \prime}$, are typically of order $\tilde{m} \gtrsim 100 \mathrm{GeV}$ [17]. Secondly, as we will see, in the quantitative discussion of Section 4, we will not need to consider gravitino masses smaller than $50 \mathrm{GeV}$.

Finally, we note that the integrated rate of the gravitino decay $\tilde{G} \stackrel{\lambda_{i j k}}{\rightarrow} \bar{\nu}_{i} \bar{e}_{j} e_{k}$ is equal to the integrated rate of the charge conjugated decay $\tilde{G} \stackrel{\lambda_{i j k}}{\rightarrow} \nu_{i} e_{j} \bar{e}_{k}$, which is given by the formula of Eq.(16).

The two kinds of gravitino decay channel involving $\lambda_{i j k}^{\prime}$ couplings are $\tilde{G} \stackrel{\lambda_{i j k}^{\prime}}{\rightarrow} \nu_{i} d_{j} \bar{d}_{k}, \bar{\nu}_{i} \bar{d}_{j} d_{k}$ and $\tilde{G} \stackrel{\lambda_{i j k}^{\prime}}{\rightarrow} e_{i} u_{j} \bar{d}_{k}, \bar{e}_{i} \bar{u}_{j} d_{k}$ (see Fig.2). Since the structures of the $\lambda_{i j k}$ and $\lambda_{i j k}^{\prime}$ interaction lagrangians are similar (see Eq.(15), Fig.1 and Fig.2), the integrated rate of the gravitino decay $\tilde{G} \stackrel{\lambda_{i j k}^{\prime}}{\rightarrow} \nu_{i} d_{j} \bar{d}_{k}\left(\tilde{G} \stackrel{\lambda_{i j k}^{\prime}}{\rightarrow} e_{i} u_{j} \bar{d}_{k}\right)$ is obtained from the formula of Eq.(16) simply by changing $\lambda_{i j k}$ to $\lambda_{i j k}^{\prime}$, adding an extra color factor of $N_{c}=3$ and replacing the masses as follows: $m_{\tilde{\nu}_{i L}} \rightarrow m_{\tilde{\nu}_{i L}}\left(m_{\tilde{e}_{i L}}\right), m_{\tilde{e}_{j L}} \rightarrow m_{\tilde{d}_{j L}}\left(m_{\tilde{u}_{j L}}\right)$ and $m_{\tilde{e}_{k R}} \rightarrow m_{\tilde{d}_{k R}}\left(m_{\tilde{d}_{k R}}\right)$. The approximation of neglecting the masses of the final state particles is also well motivated in the calculation of the gravitino decay rate involving $\lambda_{i j k}^{\prime}$ coupling constants, except when the gravitino decays into the top quark as $\tilde{G} \stackrel{\lambda_{i 3 k}^{\prime}}{\rightarrow} e_{i} t \bar{d}_{k}, \bar{e}_{i} \bar{t} d_{k}$. However, recall that in Section 4, the mass effect of the final state particles will be included in the numerical computations.

In case the gravitino decays through a coupling of type $\lambda_{i j k}^{\prime \prime}$, it decays into three quarks as $\tilde{G} \stackrel{\lambda_{i j k}^{\prime \prime}}{\rightarrow} u_{i} d_{j} d_{k}, \bar{u}_{i} \bar{d}_{j} \bar{d}_{k}$, as illustrates Fig.3. In Appendix B, we give the full analytical result for the spin summed amplitude of this gravitino decay process. In the following, we present the analytical result for the integrated rate of the gravitino decay $\tilde{G} \stackrel{\lambda_{i j k}^{\prime \prime}}{\rightarrow} u_{i} d_{j} d_{k}$, in the hypothesis the masses of the final state particles are negligible compared with the gravitino and exchanged scalar superpartner masses (see above for detailed comments on this approximation),

$$
\begin{aligned}
\Gamma\left(\tilde{G} \stackrel{\lambda_{i j k}^{\prime \prime}}{\rightarrow} u_{i} d_{j} d_{k}\right)= & \frac{N_{c} !}{96(2 \pi)^{3}} \frac{\lambda_{i j k}^{\prime \prime 2}}{m_{3 / 2}^{3} M_{\star}^{2}}\left[\Omega\left(m_{\tilde{u}_{i R}}\right)+4 \Omega\left(m_{\tilde{d}_{j R}}\right)+2 \Xi\left(m_{\tilde{u}_{i R}}, m_{\tilde{d}_{j R}}\right)\right. \\
& +2 \Sigma\left(m_{\tilde{u}_{i R}}, m_{\tilde{d}_{j R}}\right)+2 \Sigma\left(m_{\tilde{d}_{j R}}, m_{\tilde{u}_{i R}}\right)+2 \Delta\left(m_{\tilde{u}_{i R}}, m_{\tilde{d}_{j R}}\right) \\
& {\left[-S p\left(-\frac{m_{\tilde{d}_{j R}}^{2}}{m_{\tilde{u}_{i R}}^{2}}\right)-S p\left(-\frac{m_{\tilde{u}_{i R}}^{2}}{m_{\tilde{d}_{j R}}^{2}}\right)+S p\left(\frac{m_{3 / 2}^{2}-m_{\tilde{d}_{j R}}^{2}}{m_{\tilde{u}_{i R}}^{2}}\right)+S p\left(\frac{m_{3 / 2}^{2}-m_{\tilde{u}_{i R}}^{2}}{m_{\tilde{d}_{j R}}^{2}}\right)\right], }
\end{aligned}
$$

where $N_{c}=3$ is the number of colors and $\Omega(m), \Xi\left(m_{1}, m_{2}\right), \Delta\left(m_{1}, m_{2}\right)$ and $\Sigma\left(m_{1}, m_{2}\right)$ are defined in Appendix A. 


\section{Numerical results and discussion}

In this section, we present the numerical results for the gravitino decay temperature $T_{3 / 2}^{\prime}$, within the scenario characterized by an LSP gravitino having a decay channel which involves trilinear $R_{p}$ interactions. Then we will determine whether the values found for the temperature $T_{3 / 2}^{\prime}$ respect or not the limits given in Eq.(10) and Eq.(12). We will thus be able to conclude on the possibility for the scenario with an unstable LSP gravitino, decaying via $\lambda, \lambda^{\prime}$ or $\lambda^{\prime \prime}$ couplings, to solve the cosmological gravitino problem.

\section{- $\lambda_{i j k}$ couplings}

We first consider the case where one $R_{p}$ coupling constant of type $\lambda_{i j k}$ is dominant so that all the other $R_{p}$ coupling constants are negligible in comparison. The assumption of a single dominant $R_{p}$ coupling constant is often adopted in the literature (as we will remark below) for simplification reasons. This assumption find its justification in the analogy between the structures of the $R_{p}$ and Yukawa couplings, the later ones exhibiting a strong hierarchy in flavor space. In this first case, the gravitino decay temperature $T_{3 / 2}^{\prime}$ is derived (through Eq.(9)) from the gravitino decay rate involving $\lambda_{i j k}$ coupling constants $\Gamma\left(\tilde{G} \stackrel{\lambda_{i j k}}{\rightarrow} \nu_{i} e_{j} \bar{e}_{k}\right)$, which is given in Eq.(16).

In Fig.4(a) and Fig.4(b), we present the gravitino decay temperature $T_{3 / 2}^{\prime}$ as a function of the scalar superpartner mass $\tilde{m}$ for two gravitino masses, in the case of a single dominant $R_{p}$ coupling constant of type $\lambda_{i j k}$. Let us detail some particular points concerning the derivation of those numerical results. First, for simplification reason, we have set all the scalar superpartner masses to a common value, denoted by $\tilde{m}$. In order to maximize the gravitino decay rate, and thus the temperature $T_{3 / 2}^{\prime}$ (see Eq.(9)), we have also assumed that the single dominant $R_{p}$ coupling constant is $\lambda_{233}$, which is one of the $\lambda_{i j k}$ coupling constants having the weakest present low-energy constraint, namely $\lambda_{233}<0.06\left(m_{\tilde{\tau}_{R}} / 100 \mathrm{GeV}\right)[10,18]$, and that $\lambda_{233}$ is equal to its present lowenergy bound. We note that the present low-energy constraint on $\lambda_{233}$ has been obtained under the hypothesis that $\lambda_{233}$ is the single dominant $R_{p}$ coupling constant [18], which is consistent with our assumptions. At this stage, a particularity due the structure of the $\lambda_{i j k}$ couplings must be described. Since the $\lambda_{i j k}$ coupling constants are antisymmetric in the $i$ and $j$ family indices, in the considered case where $\lambda_{233}$ is a dominant $R_{p}$ coupling constant, $\lambda_{323}$ is a second dominant $\mathbb{R}_{p}$ coupling constant (having the opposite value) so that the main gravitino decay channels are $\tilde{G} \stackrel{\lambda_{233}}{\rightarrow} \nu_{\mu} \tau \bar{\tau}, \bar{\nu}_{\mu} \bar{\tau} \tau$ and $\tilde{G} \stackrel{\lambda_{323}}{\rightarrow} \nu_{\tau} \mu \bar{\tau}, \bar{\nu}_{\tau} \bar{\mu} \tau$. This point has been taken into account in the calculation. The decay temperature in Eq.(9) is proportional to the combination of statistical factors $g\left(T_{d}\right)^{1 / 4} / g\left(T_{3 / 2}\right)^{1 / 2}$. Let us here assume tentatively that the thermalized degrees of fredom at the gravitino decoupling and decay temperatures are the same as those for the minimal supersymmetric Standard Model and the present epochs, respectively. Using then the values $g\left(T_{d}\right) \simeq 915 / 4 \simeq 228.75, g\left(T_{3 / 2}\right)=43 / 11=3.909$, one obtains: $T_{3 / 2}^{\prime} \propto g\left(T_{d}\right)^{1 / 4} / g\left(T_{3 / 2}\right)^{1 / 2} \simeq 1.96$. The actual numerical value of this factor might be larger but not by very much. In the numerical results, the above ratio of statistical factors has been set to unity (we will come back to this point later). Finally, the gravitino decay rate has been multiplied by a factor of 2 in order to count the charge conjugated gravitino decay process and the masses of the final state particles have been taken into account in the computation. 
(a)

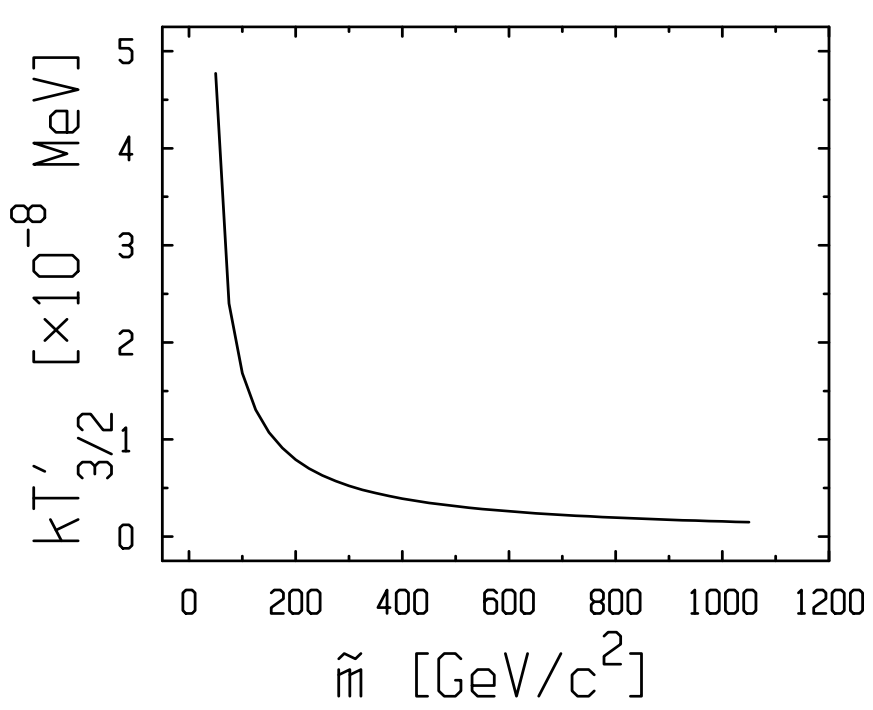

(c)

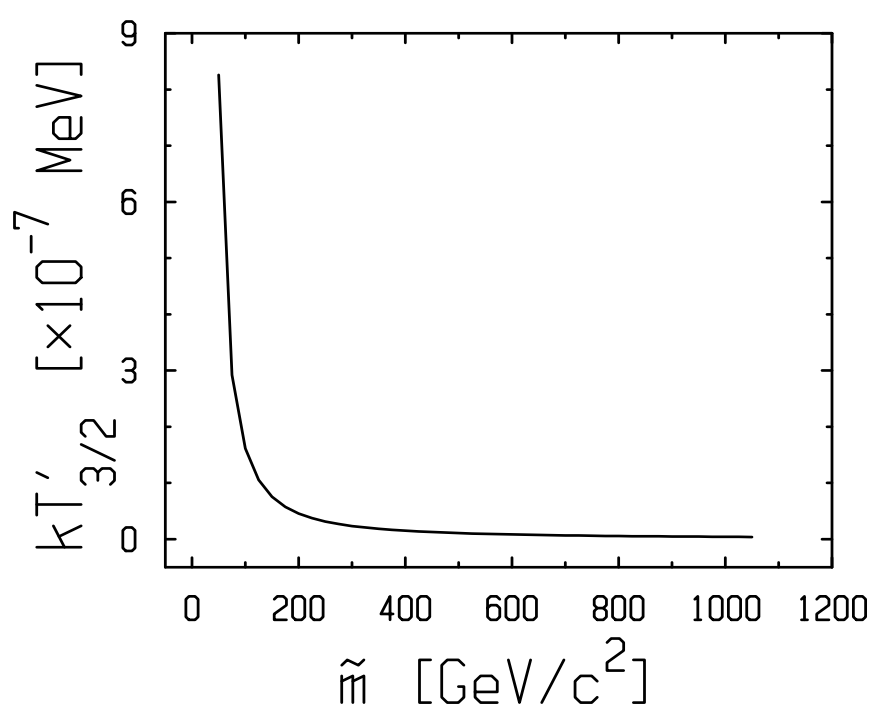

(e)

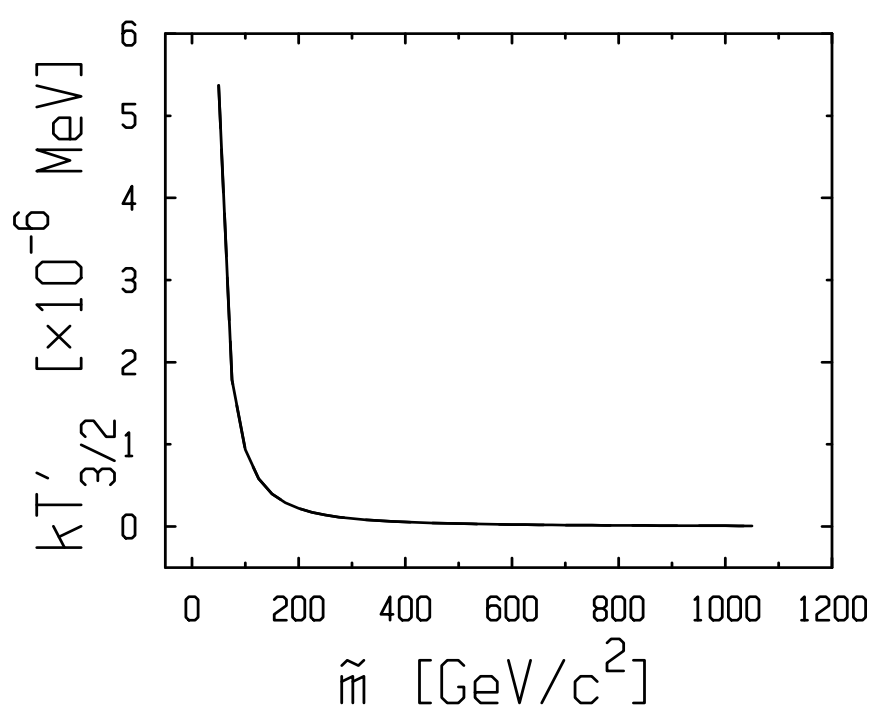

(b)

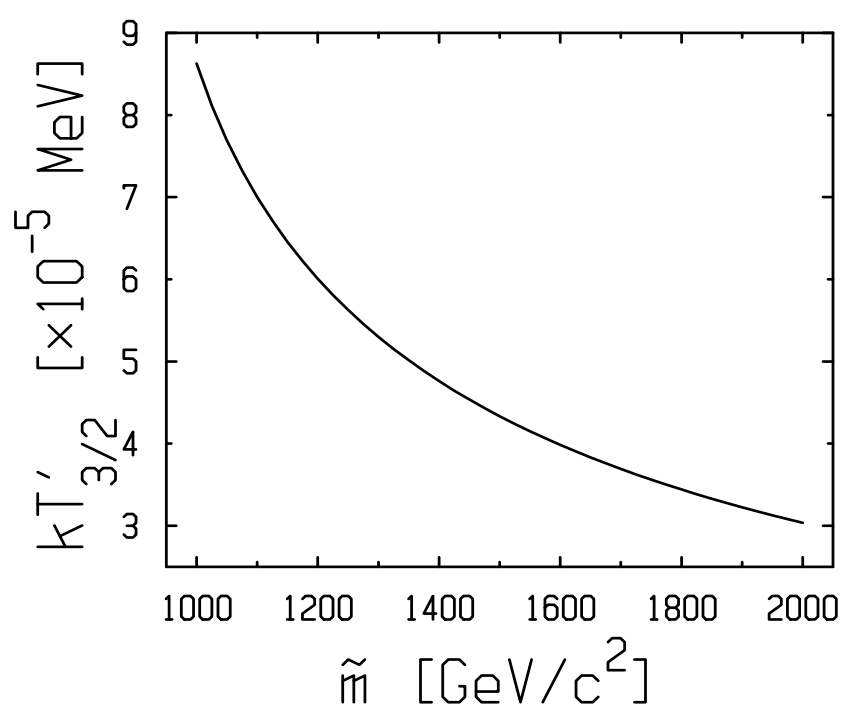

(d)

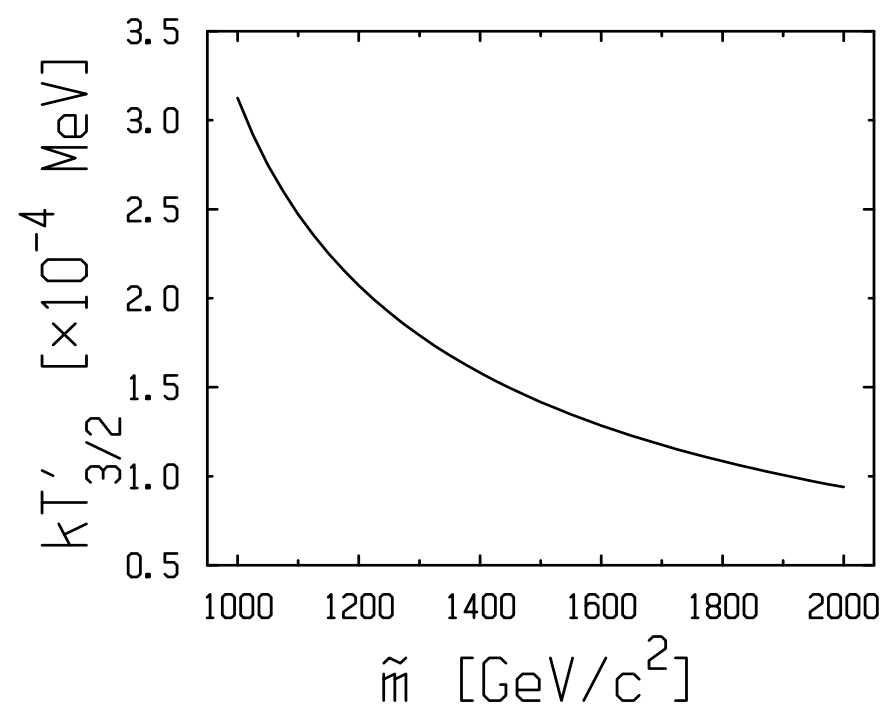

(f)

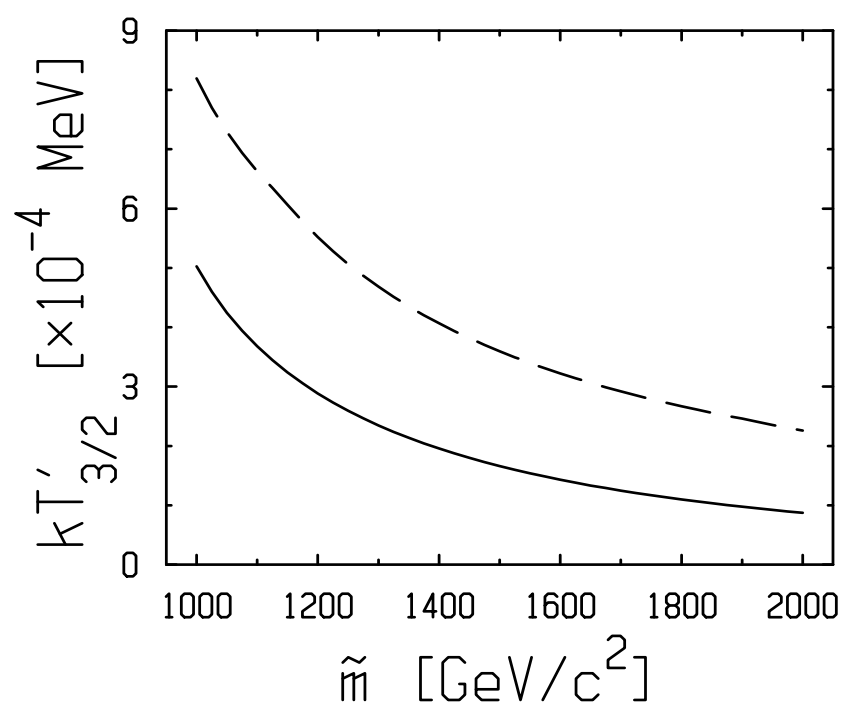


Figure 4: Gravitino decay temperature $k T_{3 / 2}^{\prime}$ (in $M e V$ ) as a function of the scalar superpartner mass $\tilde{m}$ (in $\mathrm{GeV} / \mathrm{c}^{2}$ ), with $m_{3 / 2}=50 \mathrm{GeV} / \mathrm{c}^{2}$ in Figures (a,c,e) and $m_{3 / 2}=1 T e V / c^{2}$ in Figures (b,d,f). The temperature $T_{3 / 2}^{\prime}$ represented by a plain line in Figures $(\mathrm{a}, \mathrm{b}),(\mathrm{c}, \mathrm{d})$ and $(\mathrm{e}, \mathrm{f})$ has been obtained (via Eq.(9)) from the gravitino decay rates involving the $\not R_{p}$ coupling constants $\lambda_{233}=0.06(\tilde{m} / 100 \mathrm{GeV})$, $\lambda_{331}^{\prime}=0.48$ for $\tilde{m}=100 \mathrm{GeV}$ (see [19] for the numerical dependence on $\tilde{m}$ ) and $\lambda_{213}^{\prime \prime}=1.25$, respectively. The temperature $T_{3 / 2}^{\prime}$ represented as a dashed line in Figure (f) has been derived from the sum of gravitino decay rates involving the following $\not R_{p}$ coupling constants, $\lambda_{132}^{\prime}=0.34$ for $\tilde{m}=100 \mathrm{GeV}$ (see [19] for the numerical dependence on $\tilde{m}), \lambda_{211}^{\prime}=0.09(\tilde{m} / 100 G e V), \lambda_{223}^{\prime}=0.18(\tilde{m} / 100 G e V), \lambda_{311}^{\prime}=$ $0.10(\tilde{m} / 100 \mathrm{GeV}), \lambda_{121}=0.05(\tilde{m} / 100 \mathrm{GeV})$ and $\lambda_{233}=0.06(\tilde{m} / 100 \mathrm{GeV})$.

We observe in Fig.4(a) and Fig.4(b) a decrease of the gravitino decay temperature $T_{3 / 2}^{\prime}$ when the scalar superpartner becomes heavier (particularly in Fig.4(a) for $m_{3 / 2} \sim$ $50 \mathrm{GeV}$ ). This is due to the fact that $T_{3 / 2}^{\prime}$ is proportional (see Eq.(9)) to the square root of the sum of gravitino decay rates $\Gamma\left(\tilde{G} \stackrel{\lambda_{233}}{\rightarrow} \nu_{\mu} \tau \bar{\tau}\right)+\Gamma\left(\tilde{G} \stackrel{\lambda_{323}}{\rightarrow} \nu_{\tau} \mu \bar{\tau}\right)$ which decreases as the scalar superpartner mass increases. Nevertheless, this decrease of $T_{3 / 2}^{\prime}$ when $\tilde{m}$ increases is attenuated by our choice of taking $\lambda_{233}=0.06(\tilde{m} / 100 G e V)$, since $\Gamma\left(\tilde{G} \stackrel{\lambda_{233}}{\rightarrow} \nu_{\mu} \tau \bar{\tau}\right) \propto \lambda_{233}^{2}$ and $\Gamma\left(\tilde{G} \stackrel{\lambda_{323}}{\rightarrow} \nu_{\tau} \mu \bar{\tau}\right) \propto \lambda_{323}^{2}=\lambda_{233}^{2}$ (see Eq.(16)) so that $T_{3 / 2}^{\prime} \propto \lambda_{233}$. We also remark by comparing Fig.4(a) and Fig.4(b) that $T_{3 / 2}^{\prime}$ is getting higher if $m_{3 / 2}$ is enhanced. Once more, the explanation is related to the behavior of the sum of gravitino decay rates $\Gamma\left(\tilde{G} \stackrel{\lambda_{233}}{\rightarrow} \nu_{\mu} \tau \bar{\tau}\right)+\Gamma\left(\tilde{G} \stackrel{\lambda_{323}}{\longrightarrow} \nu_{\tau} \mu \bar{\tau}\right)$ : those ones of course increase with $m_{3 / 2}$. We conclude from Fig.4(a) and Fig.4(b) that the gravitino decay temperature $T_{3 / 2}^{\prime}$ fulfills the condition 10 but not 12 , in case one $R_{p}$ coupling constant of type $\lambda_{i j k}$ is dominant. This conclusion cannot be modified by the value of the factor $g\left(T_{d}\right)^{1 / 4} / g\left(T_{3 / 2}\right)^{1 / 2}$ of Eq.(9) which is not likely to be significantly large. We must also note that only scalar superpartner masses smaller than $\mathcal{O}(\mathrm{TeV})$ have been considered in Fig.4(a) and Fig.4(b). This is to ensure that the "hierarchy problem", namely the problem of natural coexistence of the electroweak symmetry breaking scale and the scale of new physics underlying the Standard Model (grand unification scale, string scale ...), can be solved by the presence of supersymmetry. Now, if the gravitino mass (which is also the LSP mass), and thus the scalar superpartner masses, reach values as large as $\sim 10^{2} \mathrm{TeV}$, the condition of Eq.(12) can be fulfilled, in case one $R_{p}$ coupling constant of type $\lambda_{i j k}$ is dominant.

\section{- $\lambda_{i j k}^{\prime}$ couplings}

Secondly, we suppose that one $R_{p}$ coupling constant of type $\lambda_{i j k}^{\prime}$ is dominant compared with all the other $R_{p}$ coupling constants. In this framework, the temperature $T_{3 / 2}^{\prime}$ is then obtained (through Eq.(9)) from the whole gravitino decay rate, which is the sum of the two gravitino decay rates involving a $\lambda_{i j k}^{\prime}$ coupling constant, namely $\Gamma\left(\tilde{G} \stackrel{\lambda_{i j k}^{\prime}}{\rightarrow} \nu_{i} d_{j} \bar{d}_{k}\right)$ and $\Gamma\left(\tilde{G} \stackrel{\lambda_{i j k}^{\prime}}{\rightarrow} e_{i} u_{j} \bar{d}_{k}\right)$ (see Section 3$)$.

In Fig.4(c) and Fig.4(d), we show the temperature $T_{3 / 2}^{\prime}$ as a function of the scalar superpartner mass $\tilde{m}$ for two gravitino masses, in the case of a single dominant $R_{p}$ coupling constant of type $\lambda_{i j k}^{\prime}$. The explanations on the calculation of $T_{3 / 2}^{\prime}$ concerning the numerical factors and the masses of the particles involved in the gravitino decay process, which are given above for the case of a dominant $\lambda_{i j k}$ coupling, still hold in the present case. In order to optimize the temperature $T_{3 / 2}^{\prime}$, we have also supposed here that the single dominant $R_{p}$ coupling constant is $\lambda_{331}^{\prime}$, which is one of the $\lambda_{i j k}^{\prime}$ coupling constants having the 
weakest present low-energy constraint, namely $\lambda_{331}^{\prime}<0.48$ for $m_{\tilde{q}}=100 \mathrm{GeV}$ [11] (see [19] for the numerical dependence of this limit on the squark mass), and that $\lambda_{331}^{\prime}$ is equal to its present maximum allowed value. The present low-energy constraint on $\lambda_{331}^{\prime}$ has been derived by assuming that $\lambda_{331}^{\prime}$ is the single dominant $R_{p}$ coupling constant [19], which is once more consistent with our assumptions. The gravitino decay rate $\Gamma\left(\tilde{G} \stackrel{\lambda_{331}^{\prime}}{\rightarrow} \tau t \bar{d}\right)$ depends on the top quark mass that we have fixed at $m_{t o p}=174.2 \mathrm{GeV}$ [20].

The dependence of the temperature $T_{3 / 2}^{\prime}$ on $\tilde{m}$ and $m_{3 / 2}$ observed in Fig.4(c) and Fig.4(d) is similar to that seen in Fig.4(a) and Fig.4(b), which has been described above.

It appears clearly in Fig.4(c) and Fig.4(d) that the gravitino decay temperature $T_{3 / 2}^{\prime}$ respects the constraint of Eq.(10) but not of Eq.(12), in case one $R_{p}$ coupling constant of type $\lambda_{i j k}^{\prime}$ is dominant. It must be mentioned at this level that, as before, scalar superpartner masses larger than $\mathcal{O}(\mathrm{TeV})$ have not been considered in order to preserve the natural coexistence of electroweak symmetry breaking and new physics scales, guaranteed by supersymmetric theories. We have found that for gravitino masses greater than $\sim 10^{2} \mathrm{TeV}$, the constraint 12 can be respected in the case of a dominant $\lambda_{i j k}^{\prime}$ coupling.

\section{- $\lambda_{i j k}^{\prime \prime}$ couplings.}

For the case of a single dominant $R_{p}$ coupling constant of type $\lambda_{i j k}^{\prime \prime}$, the temperature $T_{3 / 2}^{\prime}$ is deduced from the gravitino decay rate involving $\lambda_{i j k}^{\prime \prime} \operatorname{coupling} \operatorname{constants} \Gamma\left(\tilde{G} \stackrel{\lambda_{i j k}^{\prime \prime}}{\rightarrow}\right.$ $u_{i} d_{j} d_{k}$ ) (see Eq.(17)).

In Fig.4(e) and Fig.4(f) (plain line), we have represented $T_{3 / 2}^{\prime}$ as a function of $\tilde{m}$ for two values of $m_{3 / 2}$, in the case of a single dominant $\lambda_{i j k}^{\prime \prime}$ coupling constant. The explanations given above on the computation of $T_{3 / 2}^{\prime}$ concerning the numerical factors, the particle masses and the anti-symmetry of the $\lambda_{i j k}$ coupling constants, still applies in the present case since the $\lambda_{i j k}^{\prime \prime}$ coupling constants are anti-symmetric in the $j$ and $k$ generation indices. We have also supposed here that the single dominant $R_{p}$ coupling constant is $\lambda_{213}^{\prime \prime}$, which is one of the $\lambda_{i j k}^{\prime \prime}$ coupling constants having the weakest present bound, namely $\lambda_{213}^{\prime \prime}<1.25$ for a supersymmetry breaking scale of $M_{S U S Y} \approx 1 \mathrm{TeV}[21,22]$, and that $\lambda_{213}^{\prime \prime}$ is equal to its present limit. Let us make a few remarks on the present bound on $\lambda_{213}^{\prime \prime}$. This constraint is not, as the previous ones, a low-energy experimental bound obtained at colliders: it originates from the requirement that $\lambda_{213}^{\prime \prime}$ remains in the perturbative domain $\left(\lambda_{213}^{\prime \prime 2} / 4 \pi<1\right)$ up to the scale of gauge group unification. We also mention that this constraint depends weakly on the value of $M_{S U S Y}$, and that it has been obtained under the hypothesis that $\lambda_{213}^{\prime \prime}$ is the dominant $R_{p}$ coupling constant.

We see in Fig.4(e) and Fig.4(f) (plain line) that the temperature $T_{3 / 2}^{\prime}$ fulfills the condition 10 but not 12 .

In Fig.5 (plain line), we show the values of the gravitino and superpartner masses for which $k T_{3 / 2}^{\prime}>0.4 \mathrm{MeV}$. These values have been obtained by assuming that the dominant $\not R p$ coupling constant is $\lambda_{213}^{\prime \prime}$ and is equal to its present limit. We see in Fig.5 (plain line) that as the superpartner mass increases, larger gravitino masses are needed to have $k T_{3 / 2}^{\prime}>0.4 \mathrm{MeV}$. The reasons are that $T_{3 / 2}^{\prime}$ increases with the gravitino mass but is suppressed if the superpartner is getting heavier (see previous figures). We can however conclude from Fig.5 (plain line) that, typically, gravitino (and scalar superpartner) masses larger than $\sim 80 \mathrm{TeV}$ are needed so that the condition of Eq.(12) can be fulfilled, in the case where $\lambda_{213}^{\prime \prime}$ is the dominant coupling constant.

Finally, we consider the situation in which a maximum number of $R_{p}$ coupling con- 


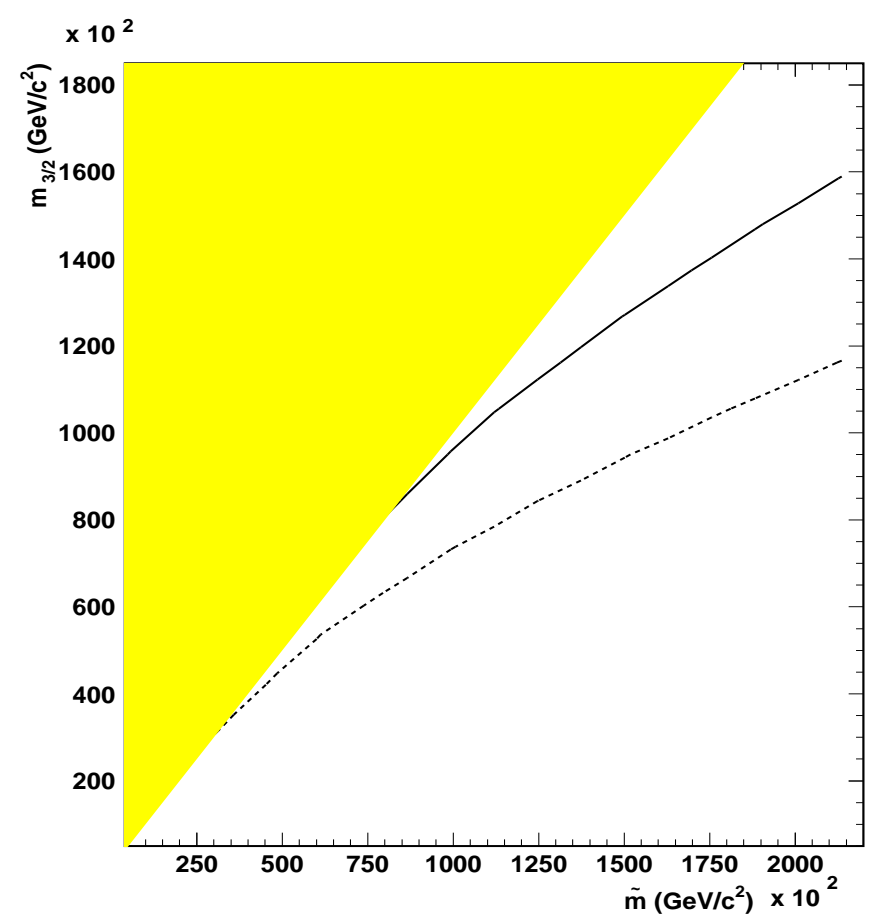

Figure 5: Domains of the $m_{3 / 2}\left(G e V / c^{2}\right)-\tilde{m}\left(G e V / c^{2}\right)$ plane (gravitino versus superpartner mass) in which the gravitino decay temperature is higher than the nucleosynthesis one, namely $k T_{3 / 2}^{\prime}>0.4 \mathrm{MeV}$. The region situated above the plain (dashed) line corresponds to $k T_{3 / 2}^{\prime}>0.4 \mathrm{MeV}$ in case the dominant $\not R_{p}$ coupling constant is $\lambda_{213}^{\prime \prime}=1.25$ (the dominant $\not R_{p}$ coupling constants are $\lambda_{132}^{\prime}=0.34$ for $\tilde{m}=100 \mathrm{GeV}, \lambda_{211}^{\prime}=0.09(\tilde{m} / 100 \mathrm{GeV}), \lambda_{223}^{\prime}=0.18(\tilde{m} / 100 \mathrm{GeV}), \lambda_{311}^{\prime}=0.10(\tilde{m} / 100 \mathrm{GeV})$, $\lambda_{121}=0.05(\tilde{m} / 100 \mathrm{GeV})$ and $\left.\lambda_{233}=0.06(\tilde{m} / 100 \mathrm{GeV})\right)$. We note that the considered $\not R_{p}$ coupling constants are fixed to the value of the perturbative limit $(\sqrt{4 \pi})$ in the mass domains where their present bound exceeds this limit. Finally, the colored region corresponds to the situation $m_{3 / 2}>\tilde{m}$ which must be considered within a scenario where the gravitino is not the LSP.

stants having the weakest present bounds are simultaneously dominant (and the considered $R_{p}$ coupling constants are equal to their present limit, as before). Based on the strongest constraints on the products of $\not R_{p}$ coupling constants and on the review [11] of the present limits on the single $R_{p}$ coupling constants, we have found that this optimistic situation corresponds to the case where the simultaneously dominant $R_{p}$ coupling constants are,

$$
\lambda_{132}^{\prime}, \lambda_{211}^{\prime}, \lambda_{223}^{\prime}, \lambda_{311}^{\prime}, \lambda_{121} \text { and } \lambda_{233}
$$

which have the present bounds $\lambda_{132}^{\prime}<0.34$ for $m_{\tilde{q}}=100 \mathrm{GeV}$ (and for instance $\lambda_{132}^{\prime} \lesssim 1.2$ for $\left.m_{\tilde{q}}=1 T e V\right)[11,19], \lambda_{211}^{\prime}<0.09\left(m_{\tilde{d}_{R}} / 100 \mathrm{GeV}\right)[18], \lambda_{223}^{\prime}<0.18\left(m_{\tilde{b}_{R}} / 100 \mathrm{GeV}\right)$ [23], $\lambda_{311}^{\prime}<0.10\left(m_{\tilde{d}_{R}} / 100 \mathrm{GeV}\right)[11,23], \lambda_{121}<0.05\left(m_{\tilde{e}_{R}} / 100 \mathrm{GeV}\right)[10,18]$ and $\lambda_{233}<$ $0.06\left(m_{\tilde{\tau}_{R}} / 100 \mathrm{GeV}\right)[10,18]$. At this stage, an important point to note is that the $\lambda_{i j k}^{\prime}$ and $\lambda_{i j k}^{\prime \prime}$ couplings cannot be simultaneously dominant, since the experimental constraints on the proton decay rate force any product $\lambda_{i j k}^{\prime} \lambda_{i^{\prime} j^{\prime} k^{\prime}}^{\prime \prime}$ to be smaller than $10^{-9}$, in a conservative way and for squark masses below $1 \mathrm{TeV}$. This result has been obtained in [26] by calculating the proton decay rate at one loop level. In contrast, we have checked here that no strong constraint exists on any product taken among the $\mathbb{R}_{p}$ coupling constants $\lambda_{132}^{\prime}$, $\lambda_{211}^{\prime}, \lambda_{223}^{\prime}, \lambda_{311}^{\prime}, \lambda_{121}$ and $\lambda_{233}$. We also note that in the optimistic situation described 
above, no other $R_{p}$ coupling constant of type $\lambda_{22 k}^{\prime}\left(\lambda_{31 k}^{\prime}\right)$ can be supposed simultaneously dominant, since the present constraint on $\lambda_{223}^{\prime}\left(\lambda_{311}^{\prime}\right)$ has been obtained under the hypothesis that $\lambda_{223}^{\prime}\left(\lambda_{311}^{\prime}\right)$ is the only dominant coupling constant among the $\lambda_{22 k}^{\prime}\left(\lambda_{31 k}^{\prime}\right)$ coupling constants [23]. This interpretation corresponds admittedly to a strong version of the single coupling constant dominance hypothesis implying that the contributions from the subdominant coupling constants do not interfere destructively in the observable used to infer the bound. Similarly, the present bound on $\lambda_{132}^{\prime}[11,19]$ (a coupling constant of type $\left.\lambda_{32 k}^{\prime}[10,24]\right)$ has been derived by assuming that it dominates over all the $\lambda_{1 j k}^{\prime}$ $\left(\lambda_{i 1 k}^{\prime}\right.$ and $\lambda_{i 2 k}^{\prime}$ ) coupling constants, so that no additional $R_{p}$ coupling constant of type $\lambda_{1 j k}^{\prime}\left(\lambda_{32 k}^{\prime}\right)$ can be assumed simultaneously dominant. Once again, if we use the present bound on $\lambda_{211}^{\prime}$, all the other $\lambda_{21 k}^{\prime}$ and $\lambda_{11 k}^{\prime}$ coupling constants must neglected [18], so that no additional $R_{p}$ coupling constant of type $\lambda_{21 k}^{\prime}$ can be considered simultaneously. $\lambda_{231}^{\prime}$ also cannot be simultaneously dominant, for another reason which is the existence of the following constraint,

$$
\lambda_{i 31}^{\prime} \lambda_{132}^{\prime}<4.710^{-5}\left(m_{\tilde{b}_{L}} / 100 \mathrm{GeV}\right)^{2}[25] .
$$

Finally, the remaining $\lambda^{\prime}$ coupling constants, $\lambda_{23 k}^{\prime}(k=2,3)$ and $\lambda_{33 k}^{\prime}(k=1,2,3)$, cannot be additional dominating couplings, because the present bounds on those have been obtained by assuming the dominance over all the $\lambda_{2 j k}^{\prime}$ and $\lambda_{3 j k}^{\prime}$ couplings, respectively $[11,19]$. Concerning the $\lambda_{i j k}$ coupling constants, the constraint on $\lambda_{121}\left(\lambda_{233}\right)$ has been obtained under the assumption that $\lambda_{121}\left(\lambda_{233}\right)$ dominates among the $\lambda_{12 k}$ and $\lambda_{11 k}^{\prime}\left(\lambda_{13 k}\right.$ and $\lambda_{23 k}$ ) coupling constants [18]. Therefore, in the optimistic framework described above, while the hypothesis that $\lambda_{121}$ and $\lambda_{233}$ simultaneously dominate remains consistent with the assumptions adopted in [18], no additional $R_{p}$ coupling constant of type $\lambda_{i j k}$ can be considered simultaneously.

In Fig.4(f) (dashed line), we have represented $T_{3 / 2}^{\prime}$ as a function of $\tilde{m}$, in the optimistic situation described above. In this situation, the temperature $T_{3 / 2}^{\prime}$ is derived from the sum of the gravitino decay rates involving the coupling constants $\lambda_{132}^{\prime}, \lambda_{211}^{\prime}, \lambda_{223}^{\prime}, \lambda_{311}^{\prime}, \lambda_{121}$, $\lambda_{211}, \lambda_{233}$ and $\lambda_{323}$.

We see in Fig.4(f) (dashed line) that the temperature $T_{3 / 2}^{\prime}$ fulfills the condition 10 but not 12. In fact, gravitino masses larger than $\sim 30 \mathrm{TeV}$ are needed so that the condition 12 can be fulfilled. This can be observed on Fig.5 (dashed line) in which have been represented the values of the gravitino and superpartner masses for which $k T_{3 / 2}^{\prime}>0.4 \mathrm{MeV}$. Those values have been derived within the optimistic scenario described above. In this optimistic scenario where several $R_{p}$ coupling constants are simultaneously present, the gravitino masses corresponding to $k T_{3 / 2}^{\prime}>0.4 \mathrm{MeV}$ are smaller than in the cases of a single dominant $R_{p}$ coupling constant, since as we have already seen $T_{3 / 2}^{\prime}$ is typically proportional to the $R_{p}$ coupling constants and decreases with the gravitino mass. It is also interesting to note that in this optimistic scenario, for a value of the ratio $g\left(T_{d}\right)^{1 / 4} / g\left(T_{3 / 2}\right)^{1 / 2}$ (see Eq.(9)) of order 50, the condition 12 is fulfilled for LSP gravitino masses of $\mathcal{O}(\mathrm{TeV})$ so that the constraint $\tilde{m} \lesssim \mathcal{O}(T e V)$ due to the hierarchy problem can be respected. However, for $g\left(k T_{3 / 2} \approx 510^{-3} \mathrm{MeV}\right) \approx 5$ (see Eq.(11)), corresponding to the value of the $S U(3)_{C} \times S U(2)_{L} \times U(1)_{Y}$ model, $g\left(T_{d}\right)^{1 / 4} / g\left(k T_{3 / 2} \approx 510^{-3} \mathrm{MeV}\right)^{1 / 2} \approx 50$ leads to $g\left(T_{d}\right) \approx 10^{8}$ which is not realistic within the present models.

In summary, if the gravitino mass, and thus the scalar superpartner masses, do not exceed the $T e V$ scale by at least one order of magnitude, the gravitino decay temperature $T_{3 / 2}^{\prime}$ fulfills well the constraint of Eq.(10) but not of Eq.(12), within the scenario of an unstable LSP gravitino decaying via $\lambda, \lambda^{\prime}$ or $\lambda^{\prime \prime}$ interactions. Hence, this scenario does 
not appear to be a natural solution to the cosmological gravitino problem.

\section{Conclusion}

Along with the existence of the cosmic microwave background, big-bang nucleosynthesis is one of the most important predictions of the big-bang cosmology. Furthermore, if one assumes that the light nuclei (atomic number less than 7 ) have effectively been produced through the big-bang nucleosynthesis, one finds that the theoretical predictions on the abundances of these light nuclei are in good agreements with the observational data [27]. Now, as we have seen above, if one believes that the light elements have been synthesized through the standard big-bang nucleosynthesis, the gravitinos must have decayed before the nucleosynthesis epoch. Therefore, since we have found that this cannot happen in the scenario characterized by an unstable LSP gravitino having a decay channel which involves trilinear $R_{p}$ interactions, this scenario does not seem to provide a realistic solution to the large relic abundance of the gravitino. It must be specified that this conclusion has been obtained by assuming that the gravitino and thus the scalar superpartner masses do not exceed the $\mathrm{TeV}$ scale so that supersymmetry can solve the so-called hierarchy problem, which is one of the strongest motivations for the existence of supersymmetry. We also mention that this final conclusion is based on the present constraints on the trilinear $R_{p}$ couplings [11] and on the assumption that the ratio $g\left(T_{d}\right)^{1 / 4} / g\left(T_{3 / 2}\right)^{1 / 2}$ takes reasonable values.

The authors of [14] have found that the lifetime of the partial two-body gravitino decay $\tilde{G} \rightarrow \gamma+\nu$, initiated by the bilinear $R_{p}$ interactions with a coupling constant yielding contributions to the neutrino mass matrix which match the observed atmospheric neutrino anomaly, reads as:

$$
\tau_{3 / 2}(\tilde{G} \rightarrow \gamma+\nu) \approx 8.310^{26}\left(\frac{1 G e V}{m_{3 / 2}}\right)^{3} \mathrm{sec}
$$

where the neutrino-photino mixing matrix element was set at the value appropriate to a photino with a mass of $80 \mathrm{GeV}$. If one assumes tentatively that the relation in Eq.(18) continues to hold in order of magnitude for the heavier gauginos case, then the condition that LSP gravitinos decay radiatively before the nucleosynthesis epoch, namely $\tau_{3 / 2} \lesssim 10^{2} \mathrm{sec}$,

requires the lower mass bound $m_{3 / 2} \gtrsim 210^{8} \mathrm{GeV}$ which conflicts with the constraint from the hierarchy problem. On the other hand, the case of light LSP gravitinos, say with mass $m_{3 / 2}<1 G e V$, leads within the bilinear $R_{p}$ option to very long lived gravitinos which could constitute a valid candidate for the dark matter [14].

Within the $R_{p}$ trilinear interactions option, by invoking the bounds on coupling constants rather than the values which match the observations involving the neutrinos mass matrix, we obtain values of the gravitino lifetime which are significantly shorter than those obtained within the bilinear interactions option by Takayama and Yamaguchi [14]. As a function of the relevant ratio of squared masses of the gravitino and exchanged sfermions, $z=m_{3 / 2}^{2} / \tilde{m}^{2}$, the predicted partial gravitino lifetime, associated to the decay into a given flavor configuration of three light fermions final state, decreases monotonically in the interval $0<z<1$ as,

$$
\tau_{3 / 2}\left(\tilde{G} \rightarrow f_{i} f_{j} f_{k}\right) \approx 10^{9}-10^{11}\left(\frac{1}{\hat{\lambda}_{i j k}^{2}}\right)\left(\frac{1 T e V}{m_{3 / 2}}\right)^{3} \mathrm{sec}
$$


where $\hat{\lambda}$ stands for any one of the trilinear coupling constants. For light gravitinos of mass $m_{3 / 2}=\mathcal{O}(\mathrm{GeV})$ and $\hat{\lambda}_{i j k}=\mathcal{O}(1)$, we see from Eq.(19) that the gravitino lifetime exceeds by a few order of magnitudes the age of the universe $t_{0} \simeq 3.210^{17} \mathrm{sec}$.

We have found that the present bounds on the trilinear $R_{p}$ couplings allow easily the gravitinos to decay before the present epoch. In consequence, the scenario of an unstable LSP gravitino having a decay channel which involves trilinear $R_{p}$ interactions could possibly solve the cosmological gravitino problem in the context of an inflationary model [28]. The reason is that within such a framework, the gravitino would not have necessarily to decay before the nucleosynthesis epoch, since in inflationary models small gravitino number densities can be predicted (for small enough gravitino number densities, the gravitino decays do not upset the nucleosynthesis). Indeed, if the universe has experienced inflationary expansion, the primordial gravitino abundance would have been completely diluted by the exponential expansion. Then, after the universe would have been reheated, gravitinos would have been regenerated by scattering processes off the thermal radiation, resulting in a secondary gravitino number density proportional to the reheating temperature, which is a parameter of the model.

\section{Acknowledgments}

We are grateful to R. Grimm and F. Takayama for helpful discussions. 


\section{A Definitions of the functions involved in the gravitino decay rates}

The mass functions $\Omega(m), \Xi\left(m_{1}, m_{2}\right), \Delta\left(m_{1}, m_{2}\right)$ and $\Sigma\left(m_{1}, m_{2}\right)$ used in Section 3 are defined in the following,

$$
\begin{gathered}
\Omega(m)=\frac{1}{48}\left(60 m^{6}-162 m^{4} m_{3 / 2}^{2}+140 m^{2} m_{3 / 2}^{4}-37 m_{3 / 2}^{6}\right. \\
\left.+12\left(m^{2}-m_{3 / 2}^{2}\right)^{3}\left(5 \frac{m^{2}}{m_{3 / 2}^{2}}-1\right) \log \left(1-\frac{m_{3 / 2}^{2}}{m^{2}}\right)\right), \\
\Xi\left(m_{1}, m_{2}\right)=\frac{1}{48}\left(84 m_{1}^{2} m_{2}^{2} m_{3 / 2}^{2}+7 m_{3 / 2}^{6}+m_{1}^{2}\left(-12 m_{1}^{4}+24 m_{2}^{4}+42 m_{1}^{2} m_{3 / 2}^{2}-40 m_{3 / 2}^{4}\right)\right. \\
+m_{2}^{2}\left(-12 m_{2}^{4}+24 m_{1}^{4}+42 m_{2}^{2} m_{3 / 2}^{2}-40 m_{3 / 2}^{4}\right)+12 m_{1}^{2}\left(\frac{2 m_{2}^{2} m_{1}^{4}-m_{1}^{6}}{m_{3 / 2}^{2}}+4 m_{1}^{4}\right. \\
\left.-4 m_{2}^{2} m_{3 / 2}^{2}-5 m_{1}^{2} m_{3 / 2}^{2}+2 m_{3 / 2}^{4}+2 m_{1}^{2} m_{2}^{2}\right) \log \left(1-\frac{m_{3 / 2}^{2}}{m_{1}^{2}}\right)+12 m_{2}^{2} \\
\left(\frac{2 m_{1}^{2} m_{2}^{4}-m_{2}^{6}}{m_{3 / 2}^{2}}+4 m_{2}^{4}-4 m_{1}^{2} m_{3 / 2}^{2}-5 m_{2}^{2} m_{3 / 2}^{2}+2 m_{3 / 2}^{4}+2 m_{1}^{2} m_{2}^{2}\right) \\
\left.\log \left(1-\frac{m_{3 / 2}^{2}}{m_{2}^{2}}\right)\right), \\
\Delta\left(m_{1}, m_{2}\right)=-m_{1}^{2} m_{2}^{2}\left(\frac{m_{1}^{2} m_{2}^{2}}{2 m_{3 / 2}^{2}}+m_{1}^{2}+m_{2}^{2}-m_{3 / 2}^{2}\right), \\
\Sigma\left(m_{1}, m_{2}\right)=\Delta\left(m_{1}, m_{2}\right) \log \left(1-\frac{m_{3 / 2}^{2}}{m_{1}^{2}}\right) \log \left(1+\frac{m_{1}^{2}}{m_{2}^{2}}-\frac{m_{3 / 2}^{2}}{m_{2}^{2}}\right),
\end{gathered}
$$

$m_{3 / 2}$ being the gravitino mass.

\section{B Formulas for the spin summed amplitudes of the gravitino decay reactions}

The full analytical result of the scared amplitude summed over the spins for the gravitino

decay reaction $\tilde{G} \stackrel{\lambda_{i j k}}{\rightarrow} \nu_{i} e_{j} \bar{e}_{k}$ is the sum of the following scared amplitude and interference terms ${ }^{1}$,

$$
\begin{aligned}
\left|M_{a}\right|^{2}= & \frac{1}{3} \frac{\lambda_{i j k}^{2}}{M_{\star}^{2}\left(m_{j k}^{2}-m_{\tilde{\nu}_{i L}}^{2}\right)^{2}}\left(m_{3 / 2}^{2}-m_{j k}^{2}+m_{\nu_{i}}^{2}\right)\left(m_{j k}^{2}-m_{e_{j}}^{2}-m_{e_{k}}^{2}\right) \\
& \left(\frac{\left(m_{3 / 2}^{2}+m_{j k}^{2}-m_{\nu_{i}}^{2}\right)^{2}}{4 m_{3 / 2}^{2}}-m_{j k}^{2}\right) \\
\left|M_{b}\right|^{2}= & \frac{1}{3} \frac{\lambda_{i j k}^{2}}{M_{\star}^{2}\left(m_{i k}^{2}-m_{\tilde{e}_{j L}}^{2}\right)^{2}}\left(m_{3 / 2}^{2}-m_{i k}^{2}+m_{e_{j}}^{2}\right)\left(m_{i k}^{2}-m_{\nu_{i}}^{2}-m_{e_{k}}^{2}\right)
\end{aligned}
$$

\footnotetext{
${ }^{1}$ The projective sums for a massive spin-3/2 field are required in order to calculate the spin averaged gravitino decay amplitudes. These projective sums are available in the literature [12, 28, 29].
} 


$$
\begin{aligned}
& \left(\frac{\left(m_{3 / 2}^{2}+m_{i k}^{2}-m_{e_{j}}^{2}\right)^{2}}{4 m_{3 / 2}^{2}}-m_{i k}^{2}\right) \\
& \left|M_{c}\right|^{2}=\frac{1}{3} \frac{\lambda_{i j k}^{2}}{M_{\star}^{2}\left(m_{i j}^{2}-m_{\tilde{e}_{k R}}^{2}\right)^{2}}\left(m_{3 / 2}^{2}-m_{i j}^{2}+m_{e_{k}}^{2}\right)\left(m_{i j}^{2}-m_{\nu_{i}}^{2}-m_{e_{j}}^{2}\right) \\
& \left(\frac{\left(m_{3 / 2}^{2}+m_{i j}^{2}-m_{e_{k}}^{2}\right)^{2}}{4 m_{3 / 2}^{2}}-m_{i j}^{2}\right) \text {, } \\
& 2 R e\left(M_{a} M_{b}^{\dagger}\right)=\frac{1}{3} \frac{\lambda_{i j k}^{2}}{M_{\star}^{2}\left(m_{j k}^{2}-m_{\tilde{\nu}_{i L}}^{2}\right)\left(m_{i k}^{2}-m_{\tilde{e}_{j L}}^{2}\right)}\left[\left(m_{i k}^{2} m_{j k}^{2}-m_{3 / 2}^{2} m_{e_{k}}^{2}-m_{\nu_{i}}^{2} m_{e_{j}}^{2}\right)\right. \\
& \left(\left(m_{3 / 2}^{2}+m_{e_{k}}^{2}-m_{\nu_{i}}^{2}-m_{e_{j}}^{2}\right)-\frac{1}{2 m_{3 / 2}^{2}}\left(m_{3 / 2}^{2}+m_{j k}^{2}-m_{\nu_{i}}^{2}\right)\right. \\
& \left.\left(m_{3 / 2}^{2}+m_{i k}^{2}-m_{e_{j}}^{2}\right)\right)+\frac{1}{2}\left(m_{i j}^{2}-m_{\nu_{i}}^{2}-m_{e_{j}}^{2}\right)\left(m_{j k}^{2}-m_{e_{j}}^{2}-m_{e_{k}}^{2}\right) \\
& \left(m_{i k}^{2}-m_{\nu_{i}}^{2}-m_{e_{k}}^{2}\right)-\frac{m_{\nu_{i}}^{2}}{2}\left(m_{j k}^{2}-m_{e_{j}}^{2}-m_{e_{k}}^{2}\right)^{2}-\frac{m_{e_{j}}^{2}}{2}\left(m_{i k}^{2}-m_{\nu_{i}}^{2}-m_{e_{k}}^{2}\right)^{2} \\
& \left.-\frac{m_{e_{k}}^{2}}{2}\left(m_{i j}^{2}-m_{\nu_{i}}^{2}-m_{e_{j}}^{2}\right)^{2}+2 m_{\nu_{i}}^{2} m_{e_{j}}^{2} m_{e_{k}}^{2}\right] \text {, } \\
& 2 R e\left(M_{b} M_{c}^{\dagger}\right)=\frac{1}{3} \frac{\lambda_{i j k}^{2}}{M_{\star}^{2}\left(m_{i k}^{2}-m_{\tilde{e}_{j L}}^{2}\right)\left(m_{i j}^{2}-m_{\tilde{e}_{k R}}^{2}\right)}\left[\left(m_{i j}^{2} m_{i k}^{2}-m_{3 / 2}^{2} m_{\nu_{i}}^{2}-m_{e_{j}}^{2} m_{e_{k}}^{2}\right)\right. \\
& \left(\left(m_{3 / 2}^{2}+m_{\nu_{i}}^{2}-m_{e_{j}}^{2}-m_{e_{k}}^{2}\right)-\frac{1}{2 m_{3 / 2}^{2}}\left(m_{3 / 2}^{2}+m_{i k}^{2}-m_{e_{j}}^{2}\right)\right. \\
& \left.\left(m_{3 / 2}^{2}+m_{i j}^{2}-m_{e_{k}}^{2}\right)\right)+\frac{1}{2}\left(m_{i j}^{2}-m_{\nu_{i}}^{2}-m_{e_{j}}^{2}\right)\left(m_{j k}^{2}-m_{e_{j}}^{2}-m_{e_{k}}^{2}\right) \\
& \left(m_{i k}^{2}-m_{\nu_{i}}^{2}-m_{e_{k}}^{2}\right)-\frac{m_{\nu_{i}}^{2}}{2}\left(m_{j k}^{2}-m_{e_{j}}^{2}-m_{e_{k}}^{2}\right)^{2}-\frac{m_{e_{j}}^{2}}{2}\left(m_{i k}^{2}-m_{\nu_{i}}^{2}-m_{e_{k}}^{2}\right)^{2} \\
& \left.-\frac{m_{e_{k}}^{2}}{2}\left(m_{i j}^{2}-m_{\nu_{i}}^{2}-m_{e_{j}}^{2}\right)^{2}+2 m_{\nu_{i}}^{2} m_{e_{j}}^{2} m_{e_{k}}^{2}\right] \text {, } \\
& 2 \operatorname{Re}\left(M_{a} M_{c}^{\dagger}\right)=\frac{1}{3} \frac{\lambda_{i j k}^{2}}{M_{\star}^{2}\left(m_{j k}^{2}-m_{\tilde{\nu}_{i L}}^{2}\right)\left(m_{i j}^{2}-m_{\tilde{e}_{k R}}^{2}\right)}\left[\left(m_{i j}^{2} m_{j k}^{2}-m_{3 / 2}^{2} m_{e_{j}}^{2}-m_{\nu_{i}}^{2} m_{e_{k}}^{2}\right)\right. \\
& \left(\left(m_{3 / 2}^{2}+m_{e_{j}}^{2}-m_{\nu_{i}}^{2}-m_{e_{k}}^{2}\right)-\frac{1}{2 m_{3 / 2}^{2}}\left(m_{3 / 2}^{2}+m_{j k}^{2}-m_{\nu_{i}}^{2}\right)\right. \\
& \left.\left(m_{3 / 2}^{2}+m_{i j}^{2}-m_{e_{k}}^{2}\right)\right)+\frac{1}{2}\left(m_{i j}^{2}-m_{\nu_{i}}^{2}-m_{e_{j}}^{2}\right)\left(m_{j k}^{2}-m_{e_{j}}^{2}-m_{e_{k}}^{2}\right) \\
& \left(m_{i k}^{2}-m_{\nu_{i}}^{2}-m_{e_{k}}^{2}\right)-\frac{m_{\nu_{i}}^{2}}{2}\left(m_{j k}^{2}-m_{e_{j}}^{2}-m_{e_{k}}^{2}\right)^{2}-\frac{m_{e_{j}}^{2}}{2}\left(m_{i k}^{2}-m_{\nu_{i}}^{2}-m_{e_{k}}^{2}\right)^{2} \\
& \left.-\frac{m_{e_{k}}^{2}}{2}\left(m_{i j}^{2}-m_{\nu_{i}}^{2}-m_{e_{j}}^{2}\right)^{2}+2 m_{\nu_{i}}^{2} m_{e_{j}}^{2} m_{e_{k}}^{2}\right] \text {, }
\end{aligned}
$$

where $M_{a}, M_{b}$ and $M_{3 / 2}$ correspond to the amplitudes of the three contributions represented in Fig.1 and marked by the same letters $a, b$ and $c$. In Eq.(B.1) to Eq.(B.6), we have used the same notations as in Section 3 and the quantities $m_{i j}, m_{j k}$ and $m_{i k}$ are defined by,

$$
m_{i j}^{2}=\left(p\left(\nu_{i}\right)+p\left(e_{j}\right)\right)^{2}
$$




$$
\begin{aligned}
m_{j k}^{2} & =\left(p\left(e_{j}\right)+p\left(e_{k}\right)\right)^{2} \\
m_{i k}^{2} & =\left(p\left(\nu_{i}\right)+p\left(e_{k}\right)\right)^{2}
\end{aligned}
$$

$p\left(\nu_{i}\right), p\left(e_{j}\right)$ and $p\left(e_{k}\right)$ being respectively the quadri-momenta of the particles $\nu_{i}, e_{j}$ and $e_{k}$. The formula for the spin summed amplitude of the gravitino decay process $\tilde{G} \stackrel{\lambda_{i j k}}{\rightarrow} \nu_{i} e_{j} \bar{e}_{k}$, which is given in Eq.(B.1)-Eq.(B.6), is equal to that of the charge conjugated process $\tilde{G} \stackrel{\lambda_{i j k}}{\rightarrow} \bar{\nu}_{i} \bar{e}_{j} e_{k}$.

The full analytical result of the scared amplitude summed over the spins for the gravitino decay reaction $\tilde{G} \stackrel{\lambda_{i j k}^{\prime}}{\rightarrow} \nu_{i} d_{j} \bar{d}_{k}\left(\tilde{G} \stackrel{\lambda_{i j k}^{\prime}}{\rightarrow} e_{i} u_{j} \bar{d}_{k}\right)$ is equal to that of the reaction $\tilde{G} \stackrel{\lambda_{i j k}}{\rightarrow}$ $\nu_{i} e_{j} \bar{e}_{k}$ given above, simply by changing $\lambda_{i j k}$ to $\lambda_{i j k}^{\prime}$, adding an extra multiplicative color factor of $N_{c}=3$ and replacing the masses as follows: $m_{\tilde{\nu}_{i L}} \rightarrow m_{\tilde{\nu}_{i L}}\left(m_{\tilde{e}_{i L}}\right), m_{\tilde{e}_{j L}} \rightarrow$ $m_{\tilde{d}_{j L}}\left(m_{\tilde{u}_{j L}}\right), m_{\tilde{e}_{k R}} \rightarrow m_{\tilde{d}_{k R}}\left(m_{\tilde{d}_{k R}}\right), m_{\nu_{i}} \rightarrow m_{\nu_{i}}\left(m_{e_{i}}\right), m_{e_{j}} \rightarrow m_{d_{j}}\left(m_{u_{j}}\right)$ and $m_{e_{k}} \rightarrow$ $m_{d_{k}}\left(m_{d_{k}}\right)$. The definitions of the quantities $m_{i j}, m_{j k}$ and $m_{i k}$ must also be changed into,

$$
\begin{aligned}
m_{i j}^{2} & =\left(p\left(\nu_{i}\right)+p\left(d_{j}\right)\right)^{2} \\
m_{j k}^{2} & =\left(p\left(d_{j}\right)+p\left(d_{k}\right)\right)^{2} \\
m_{i k}^{2} & =\left(p\left(\nu_{i}\right)+p\left(d_{k}\right)\right)^{2},
\end{aligned}
$$

for the spin summed amplitude of the reaction $\tilde{G} \stackrel{\lambda_{i j k}^{\prime}}{\rightarrow} \nu_{i} d_{j} \bar{d}_{k}$, and into,

$$
\begin{aligned}
m_{i j}^{2} & =\left(p\left(e_{i}\right)+p\left(u_{j}\right)\right)^{2} \\
m_{j k}^{2} & =\left(p\left(u_{j}\right)+p\left(d_{k}\right)\right)^{2} \\
m_{i k}^{2} & =\left(p\left(e_{i}\right)+p\left(d_{k}\right)\right)^{2},
\end{aligned}
$$

for the spin summed amplitude of the reaction $\tilde{G} \stackrel{\lambda_{i j k}^{\prime}}{\rightarrow} e_{i} u_{j} \bar{d}_{k}$. The same remark as above holds for the charge conjugated process.

The full analytical result of the scared amplitude summed over the spins for the gravitino decay reaction $\tilde{G} \stackrel{\lambda_{i j k}^{\prime \prime}}{\rightarrow} u_{i} d_{j} d_{k}$ is the sum of the following scared amplitude and interference terms,

$$
\begin{aligned}
\left|M_{a}\right|^{2}= & \frac{N_{c} !}{3} \frac{\lambda_{i j k}^{\prime \prime 2}}{M_{\star}^{2}\left(m_{j k}^{2}-m_{\tilde{u}_{i R}}^{2}\right)^{2}}\left(m_{3 / 2}^{2}-m_{j k}^{2}+m_{u_{i}}^{2}\right)\left(m_{j k}^{2}-m_{d_{j}}^{2}-m_{d_{k}}^{2}\right) \\
& \left(\frac{\left(m_{3 / 2}^{2}+m_{j k}^{2}-m_{u_{i}}^{2}\right)^{2}}{4 m_{3 / 2}^{2}}-m_{j k}^{2}\right) \\
\left|M_{b}\right|^{2}= & \frac{4 N_{c} !}{3} \frac{\lambda_{i j k}^{\prime \prime 2}}{M_{\star}^{2}\left(m_{i k}^{2}-m_{\tilde{d}_{j}}^{2}\right)^{2}}\left(m_{3 / 2}^{2}-m_{i k}^{2}+m_{d_{j}}^{2}\right)\left(m_{i k}^{2}-m_{u_{i}}^{2}-m_{d_{k}}^{2}\right) \\
2 \operatorname{Re}\left(M_{a} M_{b}^{\dagger}\right)= & \frac{\left(\frac{\left(m_{3 / 2}^{2}+m_{i k}^{2}-m_{d_{j}}^{2}\right)^{2}}{4 m_{3 / 2}^{2}}-m_{i k}^{2}\right),}{3} \frac{\lambda_{i j k}^{\prime \prime 2}}{M_{\star}^{2}\left(m_{j k}^{2}-m_{\tilde{u}_{i R}}^{2}\right)\left(m_{i k}^{2}-m_{\tilde{d}_{j R}}^{2}\right)}\left[\left(m_{i k}^{2} m_{j k}^{2}-m_{3 / 2}^{2} m_{d_{k}}^{2}-m_{u_{i}}^{2} m_{d_{j}}^{2}\right)\right. \\
& \left(\left(m_{3 / 2}^{2}+m_{d_{k}}^{2}-m_{u_{i}}^{2}-m_{d_{j}}^{2}\right)-\frac{1}{2 m_{3 / 2}^{2}}\left(m_{3 / 2}^{2}+m_{j k}^{2}-m_{u_{i}}^{2}\right)\right.
\end{aligned}
$$




$$
\begin{aligned}
& \left.\left(m_{3 / 2}^{2}+m_{i k}^{2}-m_{d_{j}}^{2}\right)\right)+\frac{1}{2}\left(m_{i j}^{2}-m_{\nu_{i}}^{2}-m_{e_{j}}^{2}\right)\left(m_{j k}^{2}-m_{e_{j}}^{2}-m_{e_{k}}^{2}\right) \\
& \left(m_{i k}^{2}-m_{\nu_{i}}^{2}-m_{e_{k}}^{2}\right)-\frac{m_{\nu_{i}}^{2}}{2}\left(m_{j k}^{2}-m_{e_{j}}^{2}-m_{e_{k}}^{2}\right)^{2}-\frac{m_{e_{j}}^{2}}{2}\left(m_{i k}^{2}-m_{\nu_{i}}^{2}-m_{e_{k}}^{2}\right)^{2} \\
& \left.-\frac{m_{e_{k}}^{2}}{2}\left(m_{i j}^{2}-m_{\nu_{i}}^{2}-m_{e_{j}}^{2}\right)^{2}+2 m_{\nu_{i}}^{2} m_{e_{j}}^{2} m_{e_{k}}^{2}\right]
\end{aligned}
$$

where $M_{a}$ and $M_{b}$ are associated to the amplitudes of the two contributions represented in Fig.3 and marked by the same letters $a, b$ and $c$. In Eq.(B.10) to Eq.(B.12), the notations of Section 3 have been used and the quantities $m_{i j}, m_{j k}$ and $m_{i k}$ are defined as follows,

$$
\begin{aligned}
m_{i j}^{2} & =\left(p\left(u_{i}\right)+p\left(d_{j}\right)\right)^{2} \\
m_{j k}^{2} & =\left(p\left(d_{j}\right)+p\left(d_{k}\right)\right)^{2} \\
m_{i k}^{2} & =\left(p\left(u_{i}\right)+p\left(d_{k}\right)\right)^{2} .
\end{aligned}
$$

The above remark on the charge conjugated process still holds. 


\section{References}

[1] H. P. Nilles, Phys. Rep. 110 (1984) 1.

[2] S. Weinberg, Phys. Rev. Lett. 48 (1982) 1303.

[3] H. Pagels and J. R. Primack, Phys. Rev. Lett. 48 (1982) 223.

[4] M. Dine, A. E. Nelson and Y. Shirman, Phys. Rev. D51 (1995) 1362.

[5] M. Dine, A. E. Nelson, Y. Nir and Y. Shirman, Phys. Rev. D53 (1996) 2658.

[6] N. Arkani-Hamed, S. Dimopoulos and G. Dvali, Phys. Lett. B429 (1998) 263.

[7] G. R. Farrar and S. Weinberg, Phys. Rev. D27 (1983) 2732.

[8] A. Salam and J. Strathdee, Nucl. Phys. B87 (1975) 85.

[9] P. Fayet, Nucl. Phys. B90 (1975) 104.

[10] H. Dreiner, published in Perspectives on Supersymmetry, Ed. by G. L. Kane, World Scientific (1998), hep-ph/9707435.

[11] G. Bhattacharyya, Nucl. Phys. B (Proc. Suppl.) 52A (1997) 83; Invited talk presented at 'Beyond the Desert', Castle Ringberg, Tegernsee, Germany, 8-14 June 1997, hep-ph/9709395.

[12] L. M. Krauss, Nucl. Phys. B277 (1983) 556.

[13] T. Banks, Y. Grossman, E. Nardi and Y. Nir, Phys. Rev. D52 (1995) 5319.

[14] F. Takayama and M. Yamaguchi, Phys. Lett. B485 (2000) 388.

[15] K. Choi, E. J. Chun and J. S. Lee, Phys. Rev. D55 (1997) 3924.

[16] K. Choi, K. Hwang and J. S. Lee, Phys. Lett. B428 (1998) 129.

[17] "Search for R-Parity Violating Decays of Supersymmetric Particles in $e^{+} e^{-}$Collisions at Centre-of-Mass Energies between $\sqrt{s}=189-208 \mathrm{GeV}$ ", ALEPH Collaboration, ALEPH 2001-012, CONF 2001-009.

[18] V. Barger, G. F. Giudice and T. Han, Phys. Rev. D40 (1989) 2987.

[19] G. Bhattacharyya, J. Ellis and K. Sridhar, Mod. Phys. Lett. A10 (1995) 1583.

[20] Top averaging group, preprint Fermilab TM-2084 (1999); D0 Collab., Phys. Rev. Lett. 79 (1997) 1197, Phys. Rev. D58 (1998) 052001, Phys. Rev. D60 (1999) 052001; CDF Collab., Phys. Rev. Lett. 80 (1998) 2767, Phys. Rev. Lett. 82 (1999) 271.

[21] B. Brahmachari and P. Roy, Phys. Rev. D50 (1994) 39; Erratum-ibid. D51 (1995) 3974 .

[22] J. L. Goity and M. Sher, Phys. Lett. B346 (1995) 69; Erratum-ibid. B385 (1996) 500 .

[23] G. Bhattacharyya and D. Choudhury, Mod. Phys. Lett. A10 (1995) 1699.

[24] K. Agashe and M. Graesser, Phys. Rev. D54 (1996) 4445. 
[25] D. Choudhury and P. Roy, Phys. Lett. B378 (1996) 153.

[26] A. Y. Smirnov and F. Vissani, Phys. Lett. B380 (1996) 317.

[27] T. P. Walker, G. Steigman, D. N. Schramm, K. A. Olive and H.-S. Kang, Ap. J. 376 (1991) 51.

[28] T. Moroi, "Effects of the Gravitino on the Inflationary Universe", Ph. D. Thesis (1995), TU-479, hep-ph/9503210, and references therein.

[29] D. Lurié, "Particles and Fields", Wiley, New York (1968). 